\title{
ARCHÉOLOGIE DES VIGNOBLES ANTIQUES DU SUD DE LA GAULE
}

\author{
Philippe BoISSINOT
}

Mots-clés. Protohistoire, époques grecque et romaine, parcellaires, plantations de vignes, traces de culture, défonçage agricole, échalas.

Key-words. Protohistory, Greek and Roman periods, field system, vineyards, traces of cultivation, land break up, vine-props.

Résumé. Avec le développement de l'archéologie de sauvetage au-delà des sites d'habitat, il est de plus en plus fréquent de rencontrer des traces de plantations antiques et préromaines. Les morphologies et les distances observées dans le Midi autorisent des interprétations en termes de viticulture et invitent à une relecture des textes agronomiques. Dans les cas les plus favorables (étendue de la fouille, faible recouvrement sédimentaire et bon contraste des sédiments), il est possible de restituer l'organisation de quelques domaines viticoles.

\begin{abstract}
With the development of rescue excavations outside urban sites, we now often find traces of pre-Roman vineyards. The morphologies and distances observed in the South allow interpretations on viticulture and a new reading of the agronomical texts. In the most favourable cases (extensive excavation, thin sedimentary covering and high contrasts of the deposits), we can make a restitution of some wineries organization.
\end{abstract}

La totalité des traces de plantation de vignes découvertes à ce jour dans le Midi de la France a été appréhendée dans le cadre de l'archéologie préventive. Dans aucun des cas, le terroir et la surface à fouiller n'ont été déterminés à l'avance pour répondre à des questions historiques ou économiques; le seul choix possible fut celui de la méthode de fouille employée et de l'horizon à décaper, suivant des contraintes de temps et d'espace assez strictes.

Dans nombre d'opérations, le contexte général des fouilles d'urgence n'a guère permis d'exploiter la totalité des faits agraires entrevus dès le décapage: à quelques exceptions près, on ignore la disposition d'éventuels échalas associés aux plantations, qui ne peuvent être révélés qu'à la suite d'une fouille fine. En outre, parce que les décapages archéologiques sont généralement menés très bas, là où les contrastes de sédiment sont les mieux marqués, on connaît mal la surface d'exploitation ancienne des vignobles, dans le cas favorable où elle est encore partiellement conservée ; en l'absence de ce point de repère, il est difficile de retrouver la généalogie des différentes façons culturales qui se sont succédé après les plantations. Enfin, le temps nous a toujours manqué pour une étude fine des impacts d'instruments agricoles visibles au fond des tranchées ou des fosses de plantation, permettant une restitution des outils employés et de la gestuelle associée.

Le succès d'un décapage (à savoir, la mise en évidence de traces et de dispositifs sur une grande étendue) dépend du contraste entre les sédiments remués par les anciens agriculteurs et ceux restés vierges de toute exploitation, soit un contexte géomorphologique indépendant et antérieur aux façons culturales que nous devons analyser (Boissinot, 1997; Boissinot, Brochier, 1997). Ce contexte doit être préalablement explicité par l'archéologue, avant que les traces agraires, considérées comme des anomalies, soient mises en évidence. Dans le Midi, la période qui nous intéresse ici, qui s'étend de la fin de l'âge du Fer à l'Antiquité tardive, a livré des indices souvent inscrits dans de tels contextes favorables, les fosses de plantation au remplissage 
plus clair venant profondément inciser les premiers sols bruns de l'Holocène (Boissinot, à paraître). On ne peut en dire autant des vestiges postérieurs (médiévaux et modernes), généralement noyés dans des formations sédimentaires peu contrastées, qui ont souvent fait les frais des exploitations agricoles intensives des deux derniers siècles ${ }^{101}$.

Une fois mises en évidence sur de grandes surfaces, que faire de ces anomalies du sol aux contours si géométriques ? Des considérations sur la disposition, les espacements et l'énergie mobilisée pour les creusements ${ }^{102}$ permettent d'écarter les productions agricoles annuelles et orientent rapidement vers l'arboriculture. Les preuves palćobotaniques de la plantation de la vigne dans ces fosses et fossés existent désormais, mais ne sont pas à ce jour publiées; nous nous contenterons donc ici des fortes présomptions formulées dès les premières découvertes de Saint-Jean-du-Désert, Lunel-Viel et Nîmes au début des années 1990 (Fiches, 1996). À ce jour, aucun élément contradictoire n'est venu remettre en cause les caractères généraux de ces interprétations initiales. Maintenant que quelques exemples démonstratifs sont publiés (Vidal, Petitot, 1992 ; Favory et al., 1993; Boissinot, 1995a; Monteil et al., 1999), les découvertes de plantation antiques et protohistoriques se multiplient, parfois en association avec des structures liées à la vinification. Aux premiers décapages exigus, souvent limités à quelques tranchées permettant d'observer la morphologie des creusements et leurs espacements, succède maintenant une approche spatiale des domaines antiques, intégrant sur des surfaces que l'on souhaiterait les plus vastes possible des données concernant l'habitat, les nécropoles, les chemins, les fossés et les traces de culture. Ce type d'expérience a été tenté au cours de deux fouilles préventives: aux Girardes (Lapalud, Vaucluse) dans le

101. Pour ces raisons préciscs, il a ćtć impossible de mettre en évidence quelque plantation que ce soit sur le site des Plantades à Gerzat (Puydc-Dômc), pour lequel les archives sont pourtant très explicites. L.a fouille, particulièrement attentive aux problèmes agraires, n'a pas permis de retrouver les fosses de plantation de vignes attendues à l'intérieur d'un systc̀me fossoyć bien matérialisé (Boissinot, inédit).

102. Exemple de calcul pour Dassargues à Lunel, (Hérault) : afin de bonifier le terrain, $500 \mathrm{~m}^{3}$ de terre ont été remués pour chaque surface d'une jugère, ce qui représente un mois ct demi de travail pour une équipe de cinq ouvriers selon des estimations modernes (Garnier et al., 1995 , p. 34). Les auteurs pensent qu'une telle énergie mobilisée excèdc les capacités d'une initiative individuelle ou familiale. cadre de l'opération TGV-Méditerranée ${ }^{103}$ et à La Quintarié (Clermont-l'Hérault, Hérault) sur le tracé de l'autoroute A $75^{104}$, toutes deux concernant des surfaces dépassant les 20 ha appréhendées dans leur contexte géoarchéologique. À côté de l'approche extensive des terroirs viticoles, plusieurs fouilles plus réduites ont livré des résultats intéressants: dans la périphérie de Nîmes préromaine et romaine, les traces de cultures ont depuis longtemps déjà attiré l'attention des archéologues ${ }^{105}$. Proches de la ville ou recouverts à un moment donné par l'expansion urbaine, les vestiges de champs y sont particulièrement bien conservés et remarquablement datés (exemple du Florian, cf. Monteil et al., 1999). Enfin, aux abords de l'agglomération de Lattes, de nouvelles découvertes permettent désormais de visualiser les vignobles que l'on soupçonnait à partir d'autres données (Buxó i Capdevila, 1992 ; Py dir., 1992 ; Puertas, 1998).

Nous nous livrerons dans ce qui suit à un va-et-vient incessant avec les textes des Agronomes ${ }^{106}$ de l'Antiquité, non pour illustrer sur le terrain les propos des anciens, mais pour envisager toute la complexité de l'interprétation des traces ou éclaircir certains passages obscurs des écrits canoniques. Chaque fois que cela sera possible, on envisagera les observations sous l'angle technologique des chaînes opératoires (Amouretti, 1988).

Les exemples choisis pour illustrer notre propos se placent essentiellement dans le Midi de la France (Narbonnaise), où la recherche sur les parcellaires et les paysages ruraux est depuis longtemps bien développée, avec un souci plus récent d'intégrer les données géoarchéologiques. D'autres découvertes similaires sont signalées dans le Centre-Ouest (Barbezieux-Saint-Hilaire, Charente) ; les fouilles récentes de Wollaston en Angleterre (Meadows, 1998) laissent supposer des trouvailles encore plus septentrionales.

103. Sous la direction de P. Boissinot et K. Roger. Au cours de la même opération (TGV-Méditerranée), plusicurs fouilles plus réduites ont été entreprises dans les Bouches-du-Rhône sur le thème spécifique des " traces agraircs" dans la diachronie (direction P. Boissinot). Ce programme visait à établir la généralité des faits agraires dans les formations holocènes ainsi qu'un corpus des formes rencontrées.

104. Sous la direction de P. Boissinot et H. Pomarèdes.

105. Sous l'impulsion de P. Poupet et d'autres chercheurs nîmois, dans le cadre initial d'un projet d'archéologie spatiale dit Nîmes rurbain.

106. Que nous appellerons désormais les Agronomes (avec une majuscule) pour simplifier la présentation. 


\section{LES PLANTATIONS}

\section{EMPLACEMENT}

Lorsqu'on veut établir un vignoble, la première question qui se pose est celle de son implantation topographique. Tout en formulant leurs préférences - généralement un coteau bien ensoleillé et découvert -, les Agronomes envisagent toutes les possibilités, que le terrain soit dans une vallée étroite (depressio), sur une colline aux pentes raides (collis), sur un versant (acclivitas) ou dans une plaine (campus) dont les tendances peuvent être parfois marécageuses (palus).

Les découvertes archéologiques faites en Gaule Narbonnaise se ramènent pour l'instant aux trois derniers cas. Lorsque le sous-sol est argileux et draine mal les eaux souterraines, comme c'est le cas à SaintJean-du-Désert, des tranchées remplies de pierres sont effectuées avant la plantation. On sait d'après Columelle (De l'agriculture, II, 2) et Pline (H. N., XVII, 3) que, durant l'Antiquité, les marais Pontins et les marécages de Ravenne portaient des vignobles florissants grâce aux opérations de drainage (Billiard, 1913, p. 247).

\section{PRÉCÉDENTS CULTURAUX}

La question du précédent cultural est un thème récurrent des recommandations aux viticulteurs. Lorsqu'une vigne doit succéder à une autre vigne, il faut attendre longtemps et prendre la peine d'expurger les anciennes racines afin de détruire le poison (venenum) qui engourdit la terre (Columelle, De l'agriculture, III, 11, 2). Avec des arguments quelque peu différents, les agronomes modernes ou contemporains réclament des précautions similaires.

Dans le parcellaire préromain de Saint-Jean-duDésert, les cas de successions culturales sont rares et il est peu probable que les traces les plus récentes relèvent aussi de la viticulture. À La Quintarié, sur treize plantations reconnues, une seule présente une configuration complexe avec des fosses qui ne peuvent pas être toutes attribuées à la technique du provignage; pour cette raison, nous retiendrons la possibilité d'une succession d'au moins deux vignobles. À Nîmes, sur le site du Florian, au moins trois phases de plantations, toutes de morphologies différentes, se succèdent entre 125-100 avant J.-C. et 40-30 avant J.-C., avec quelques décalages cependant (Monteil et al., 1999). Si l'on répartit régulièrement dans l'intervalle chronologique proposé par les fouilleurs les trois moments consacrés aux plantations, on obtient des temps de repos supérieurs à cinq ans, durée minimale proposée par Sinety au début du XIX' s. (Pellicot, 1866, p. 165).

En ce qui concerne un éventuel précédent cultural, on ne possède pour l'instant aucune observation archéologique démontrant sans ambiguïté l'enlèvement de souches ou de racines anciennes, action matérialisée par la déformation des structures de plantation initiales. D'une manière générale, on ne s'est guère posé le problème de la préparation du terrain préalable aux défoncements, opérations de défrichement pourtant décrites avec détail dans les ouvrages des Agronomes. Il est vrai que l'on ignore souvent la surface à partir de laquelle les fosses ou les fossés ont été creusés et que l'urgence des opérations conduites jusqu'à présent ne laisse guère la possibilité de s'attarder sur les formes (creusements, perturbations) les plus complexes.

\section{DÉFONCEMENTS}

Il n'est pas toujours aisé de distinguer entre défoncement et plantation, quoique en théorie l'un précède nécessairement l'autre. Parfois, la première opération qui vise à ameublir et retourner le sol pour le rendre perméable à l'action de l'atmosphère, des pluies et du soleil, n'est accomplie que partiellement sur la surface à planter. Columelle donne l'exemple d'un vignoble où seulement la moitié des rangées a fait l'objet d'un défoncement préliminaire, les lignes intercalaires étant obtenues par marcottage grâce à des fosses peu profondes, qui ne bénificient pas à leur emplacement des avantages du défoncement (Columelle, De l'agriculture, III, 13, 5). Nous avons tenté de traduire sur la figure 5 le procédé décrit par l'auteur, qui correspond probablement aussi aux observations de terrain faites dans le champ $a$ de Saint-Jean-duDésert (fig. 6). Ce cas nous montre en outre que le plan d'un vignoble n'est pas forcément réalisé au cours d'une seule étape et que toute une chronologie peut être décrite.

Lorsque l'implantation du vignoble se réduit au creusement de fosses isolées, il est clair que défoncement et 


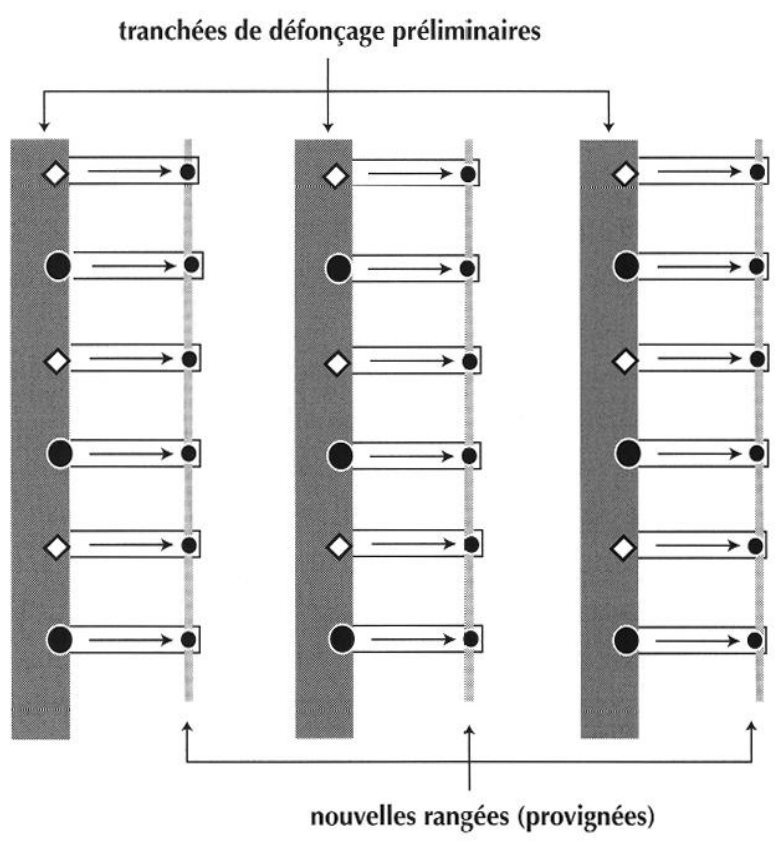

\begin{tabular}{|lll|}
$\longrightarrow$ & provignage & plants vifs initiaux \\
$\diamond$ & mailletons & ceps obtenus par provignage \\
\hline
\end{tabular}

Fig. 5 - Essai de représentalion graphique d'un champ partiellement défoncé et complélé par provignage d'après la descriplion donnée par Columelle (De l'agriculturc, III, 13, 5).

plantation se confondent dans un volume réduit, minimisant ainsi les coûts des travaux.

Les Agronomes distinguent en général trois types de défoncement (pastinatio, depastinatio): de manière uniforme, par tranchées et par fosses.

\section{DÉFONCEMENT UNIFORME}

Cette technique, de loin la plus performante, est surtout recommandée lorsqu'on doit rcprendrc un ancien vignoble ou défricher un bois. Le terrain est alors entièrement retourné à la main avec des outils tels que le bipalium, la pala et le rutrum, en progressant suivant des tranchées régulières, en mettant la terre derrière soi et en prenant soin de ne laisser aucune banquette non retournée (Columelle, De l'agricullure, III, 13, 9). Les profondeurs indiquées sont étonnantes : 2,5 pieds $(73,9 \mathrm{~cm})$ dans les plaines, 3 pieds $(88,7 \mathrm{~cm})$ dans les terrains en pente et jusqu'à 4 pieds $(118,2 \mathrm{~cm})$ sur les versants plus abrupts des collines où l'auteur a conscience de l'ampli-

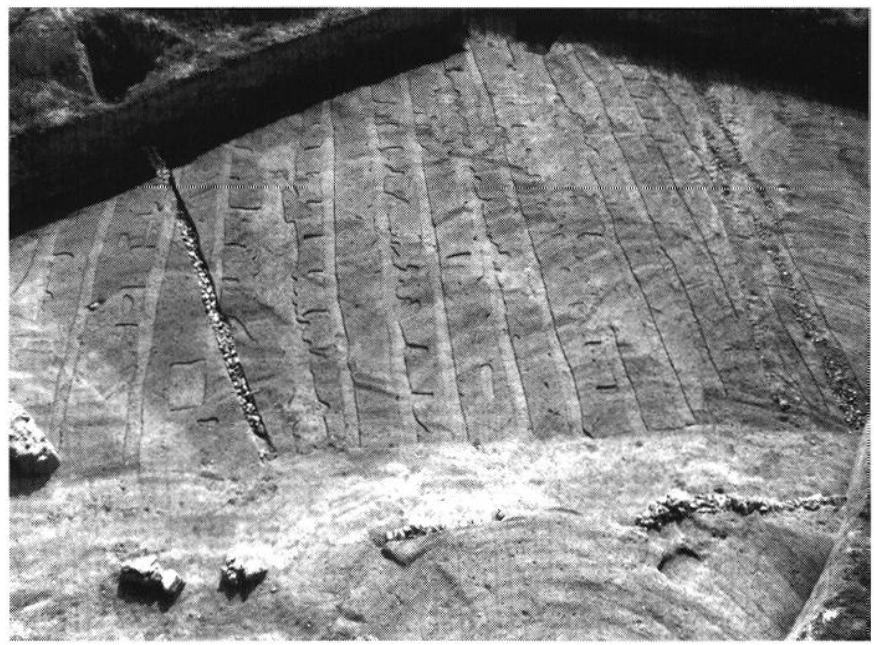

Fig. 6 - Vue vers le nord-est du champ a de Saint-Jean-du-Désert à Marseille (époque hellénistique), constitué d'une série de tranchées avec plusieurs creusements transversaux indiquant la pratique du provignage. À gauche, tranchée remplie de cailloux permeltant le drainage du terrain; en bas, ruisseau fossile colmaté par une crue à l'époque romaine.

fication des phénomènes d'érosion (fig. 7). Ces profondeurs sont à peine supérieures à celles préconisées par la Statistique de Villeneuve au début du XIX ${ }^{\mathrm{e}} \mathrm{s}$. (1829, IV, p. 268) dans laquelle on dénombre cinq techniques principales mettant en œuvre, isolément ou de manière combinée, la charrue, la bêche et la houc. À cette époque, les défoncements des plaines de l'Arc (Bouchesdu-Rhône) destinées à la vigne atteignent une profondeur maximum de $55 \mathrm{~cm}$; on note cependant que des défoncements plus coûteux, réalisés par trois ouvriers selon la technique de «l'effondrement à la Marseillaise » (Pellicot, 1866, p. 169; Blanchemanche, 1990, p. 23), permettaient d'ameublir le sol sur environ $1 \mathrm{~m}$ de profondeur. Il est difficile d'observer des horizons aussi épais en fouille pour au moins une raison : si des creusemonts aussi importants ont été réalisés, il est peu probable que l'ensemble ainsi homogénéisé ait été conservé intact jusqu'à nous, sans incisions supcrficielles ou remaniements partiels dus à des labours postérieurs. En outre, la plupart des décapages archéologiques étant préalablement réalisés à la pelle mécanique, il y a peu de chances pour que la fouille révèle autre chose qu'une interface à peine bosselée, comportant encore dans le meilleur des cas les traces d'impacts des outils agricoles. Pour ces raisons, nous n'avons aucun exemple de cette technique antique dans le Midi de la France, où 


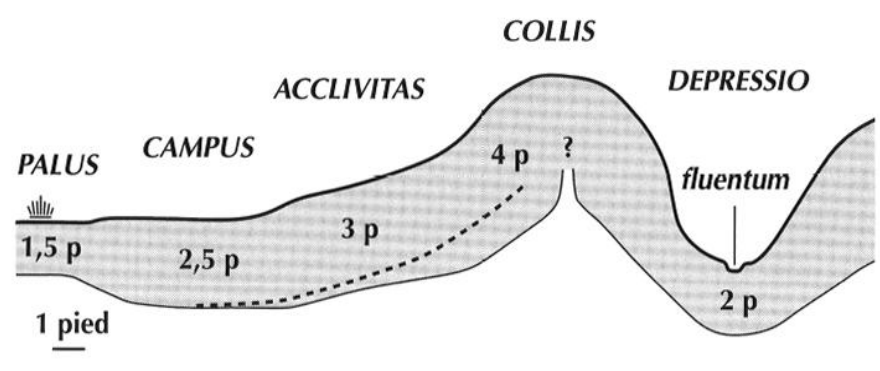

Fig. 7 - Profondeur de défoncement suivant la nature du terrain d'après le livre III de Columelle (en gris); le trait discontinu indique la profondeur des plantations.

d'ailleurs elle aurait été ignorée si l'on en croit Columelle.

\section{DÉFONCEMENT PAR TRANCHÉES (SULCI)}

Cette technique permet une évidente économie d'énergie, tout en résolvant le problème du drainage dans les zones basses. Cependant, les parois des tranchées se comportant comme des murs de terre, le procédé s'avérait néfaste au bon développement racinaire des plantes (Billiard, 1913, p. 256). La plantation s'effectuant dans l'axe de la tranchée à une profondeur inférieure à celle du défoncement, seules les éventuelles fosses (transversales) de provignage peuvent nous livrer l'emplacement des ceps initiaux. Dans la plupart des cas, l'archéologue doit se contenter de la morphologie et de l'écartement des tranchées, sans possibilité de restitution précise du vignoble.

Cette technique de défoncement a été mise en évidence sur plusieurs sites du Midi de la France datant de l'âge du Fer ou de la période romaine: à Nîmes (Le Florian, Mas Carbonnel, Le Viol-du-Plan, SaintAndré-de-Codols), Marseille (Saint-Jean-du-Désert, Alcazar), Sauvian (La Domergue) et Ventabren (Les Bosques).

Dans l'exemple de Saint-Jean-du-Désert, un seul champ (fig. 6) montre l'usage des tranchées que l'on peut estimer à une cinquantaine (Boissinot, 1995b, p. 37). Leur largeur moyenne est de $0,40 \mathrm{~m}$ et leurs entraxes varient entre $1,10 \mathrm{~m}$ et $1,20 \mathrm{~m}$. Ces dimensions diffèrent nettement de celles que l'on peut lire dans le traité de Columelle, qui préconise des tranchées de 6 pieds de largeur séparées par des espaces trois fois plus grands, dans le cas où on cherche à imiter partiellement un défoncement (Columelle, De l'agriculture, III, 13, 4). Même dans le cas déjà cité d'un vignoble à deux temps, mettant en place des plants vifs, des mailletons et des marcottes (fig. 5), la largeur des tranchées initiales est de 5 pieds, dimension toujours supérieure à nos données de terrain. De même que les valeurs, le rapport entre l'espace laissé vierge et celui défoncé ne coincide pas non plus. Compte tenu des dimensions proposées par Columelle, il pourrait s'agir d'une culture sur deux rangs, les deux files de ceps étant placées dans de larges tranchées de défoncement et l'espace intercalaire dévolu à d'autres cultures, comme cela était le cas dans les oullières de Provence des XVIII" et XIX ${ }^{\mathrm{e}} \mathrm{s}$. On sait que durant l'Antiquité les Ombriens et les Marses cultivaient du blé dans des planches (porculeta) entre les vignes, sur une largeur pouvant atteindre 20 pieds (Pline, H. N., XVII, 171).

Les quelques découvertes de tranchées de défoncement en Italie (Bradford, 1950 ; Broise, Jolivet, 1995; Calci, Sorella, 1995) s'accordent mieux avec les propositions des Agronomes : les tranchées sont en général moins étroites ( $0,9 \mathrm{~m}$ en moyenne) et les espaces intercalaires ont des largeurs qui dépassent souvent les $5 \mathrm{~m}$ (Musarna, Macchia del Conte, Asinello, Acqua Acetosa, Monte dello Spavento, Via Casal Bianco). S'agitil là de façons culturales propres à l'Italie pour lesquelles les Agronomes auraient pu disposer d'une documentation mieux fournie ? Faut-il envisager dans ces contrées une préférence pour les vignes avec cultures intercalaires? Ou bien, doit-on invoquer le hasard des découvertes et des séries archéologiques encore peu significatives?

D'une manière générale, les traces observées en Narbonnaise montrent des largeurs de tranchées nettement inférieures: de $0,20 \mathrm{~m}$ à $0,40 \mathrm{~m}$ en moyenne. Quant aux espacements de bord à bord, ils varient de $0,8 \mathrm{~m}$ (Saint-Jean-du-Désert) à $2,75 \mathrm{~m}$ (Le Florian). Avec des espacements variant entre $0,55 \mathrm{~m}$ et $0,65 \mathrm{~m}$, le cas du Mas Carbonnel aux portes de Nîmes fait figure d'exception.

Le comblement des tranchées se présente généralement de manière homogène, avec indices de bioturbation et sans traces de ruissellement. Ces caractères observés aussi bien à Saint-Jean-du-Désert qu'au Florian indiquent la rapidité du rebouchage des excavations. La mauvaise représentation de la fraction caillouteuse au sein du remplissage indique certainement la pratique d'un épierrement préliminaire, que l'on peut mettre en 


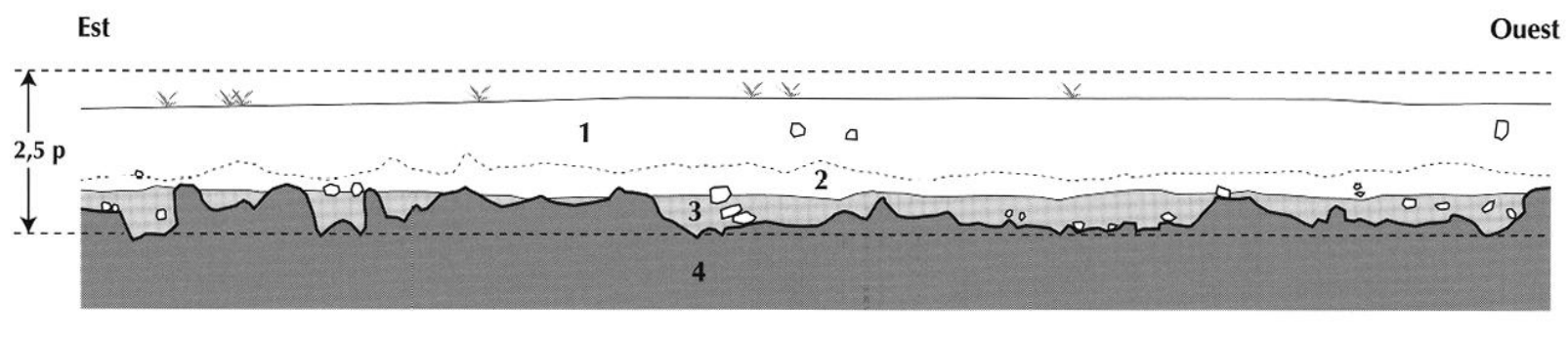

0 $1 \mathrm{~m}$

Fig. 8 - Relevé stratigraphique dans le champg des Girardes à Lapalud (Haut-Empire). La couche 4 consitue l'encaissant et la couche 3 le remplissage des différenles structures agraires de cette période. Nous avons indiqué dans la marge la profondeur minimale de 2,5 pieds préconisée par les Agronomes antiques.

relation avec les recommandations de Columelle dans le livre II de son traité (De l'agricullure, II, 2) ${ }^{107}$. L'apport d'engrais organique est plus difficile à démontrer: les tessons érodés retrouvés dans le remplissage des tranchées peuvent aussi bien provenir de la plantation en question que d'une culture antérieure.

En ce qui concerne les profondeurs requises - de 3 pieds en général à 2,5 pieds dans le cas d'un défoncement à l'économie sclon Columelle -, les données de terrain livrent des valcurs nettement inféricures. À SaintJcan-du-Désert, les tranchées sont profondes d'à peine plus de 2 pieds si l'on compte à titre d'hypothèse $0,30 \mathrm{~m}$ de terre labourée au-dessus du sol noir sous-jacent (Boissinot, 1995b). Il s'agit là pourtant des creusements les plus profonds découverts dans le Midi. Au Florian, la profondeur des tranchées d'époque républicaine est plus proche de la moyenne observée : $0,40 \mathrm{~m}$ minimum si l'on additionne les $0,20 \mathrm{~m}$ lisibles dans l'encaissant à l'épaisseur du paléosol brun-rouge associć à la culture (Montcil et al., 1999).

Ce que l'on remarque sur les fossés peut être aussi formulé pour les fosses, que nous allons maintenant évoquer. Nous avons indiqué sur l'un des relevés stratigraphiques des Girardes quel devait être le niveau du sol si les plantations avaient été creusées jusqu'à 2,5 pieds,

107. Certaines fosses remplics de pierres découvertes au scin des traces agraires de Château-Blanc à Ventabren (općration TGV-Méditerranćc) pourraient correspondre à l'une des techniques d'épierrement prćconisée par Columelle : "s'il y a unc grande quantité (de pierres), on les rassemblera en tas dans une partie du champ, ou bien encore on les enterrera dans une tranchéc profonde " (Columelle, De l'agriculture, II, 2) ; cependant, dans le cas précis de ce site, l'absence de toute relation stratigraphique ne nous permet pas d'assurer une datation antique de ces structures. comme Columelle le recommande pour les champs en plaine (fig. 8). On constate que l'ensemble du remplissage holocène n'atteint pas la puissance attendue; compte tenu de la planćité de cette partie du Tricastin, il est peu probable que le bilan sédimentaire se soit fait ici en faveur de l'érosion. On en concluera donc que les creusements opérés n'ont pas atteint la profondeur préconisée par Columelle. On notera d'ailleurs que les agronomes méridionaux du $\mathrm{XIX}^{\mathrm{c}} \mathrm{s}$. recommandaient plutôt des profondeurs comprises entre $0,30 \mathrm{~m}$ et $0,60 \mathrm{~m}$ (Pellicot, 1866, p. 183), valeurs plus proches de nos données de terrain.

\section{DÉFONCEMENT PAR FOSSES ISOLÉES (SCROBES)}

Cette technique est de loin la plus économique car elle ne concerne qu'un tout petit volume autour du plant. Elle présente toutefois deux inconvénients (Billiard, 1913, p. 255) : d'une part, celui de limiter la première croissance du plant, surtout dans les terrains compacts, et, d'autre part, celui de se comporter comme une citerne en cas de pluies fréquentes et de sol imperméable. Sur ces questions, la littérature antique est abondante et les solutions pour y remédier très variées. Il en résulte une grande dispersion des mesures proposécs ; en dehors de quelques formules à l'évidence recopiées, on ne trouve pas deux auteurs pour proposer un même écartement de fosses, un rapport identique entre longueur et largeur (Billiard, 1913, p. 300-301).

La seconde cause de variation concerne la nature et le nombre de plants installés dans la fosse. Pour Columelle, les mailletons sont placés dans des fosses de 3 pieds de longueur et de 2 pieds de profondeur " avec une aussi 


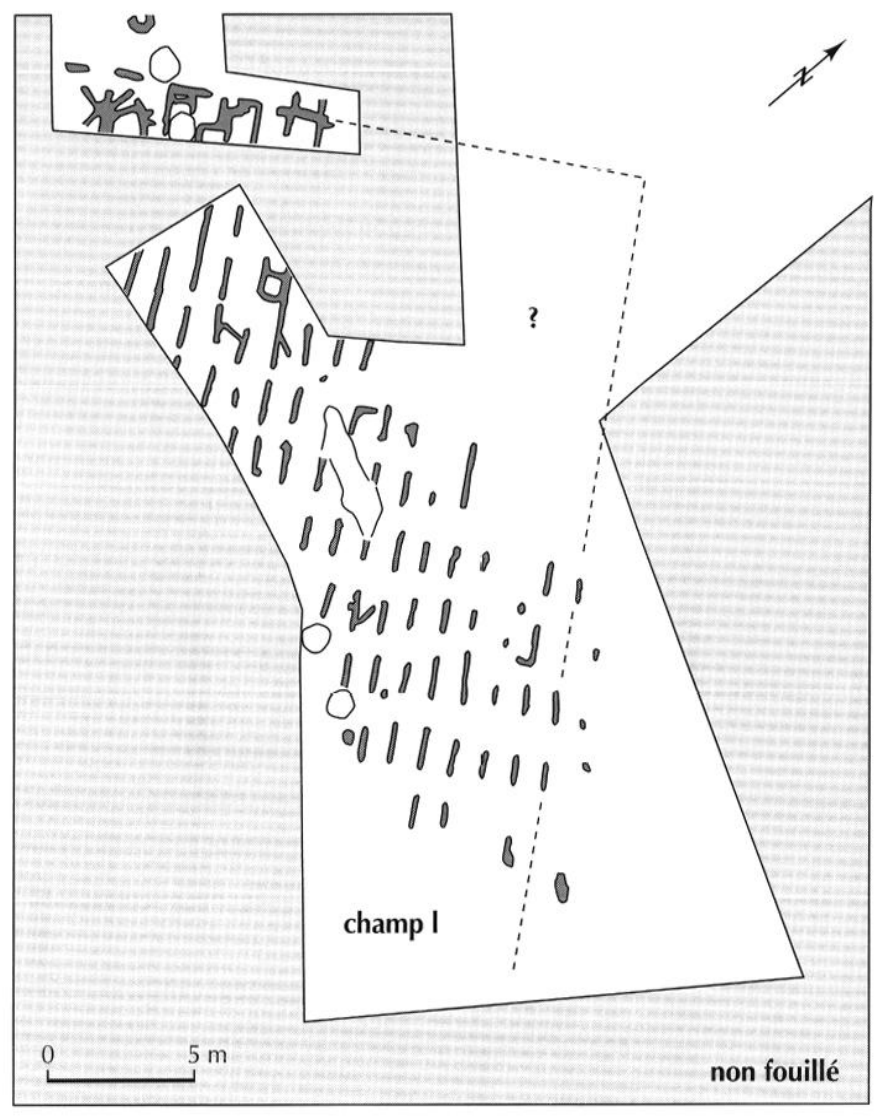

Fig. 9 - Plan de l'extrémité nord-est du champ de plantation 1 de La Quinlarié à Clermont-l'Héraull (Haul-Lmpire) constitué de fosses allongées de type alvei.

grande largeur que permet celle de l'outil. [...]. [Les viticulteurs] font ces opérations en suivant un même cordeau et en laissant entre les fosses des banquettes de même nombre de pieds jusqu'à ce qu'ils terminent leur rangée. Ayant laissé un espace, ils entreprennent alors une nouvelle rangée avec, suivant la façon de faire de chaque cultivateur, soit le hoyau, soit la charrue. Et si la terre est retournée seulement par un homme chargé de creuser, l'intervalle entre les rangées est au moins de cinq pieds, au plus de sept pieds ; mais si c'est par une charrue et des bœufs, le minimum est sept pieds, dix font une bonne largeur " (Columelle, De l'agriculture, III, 13, trad. Dumont éd., 1993, p. 56-57). Si on laisse de côté la précision métrologique, il est possible de faire des comparaisons avec les vignobles mis en évidence à La Quintarié, faits de segments disposés en rangées dont la largeur à peine inférieure à $0,20 \mathrm{~m}$ est sans doute très proche de celle de l'instrument utilisé (fig. 9).

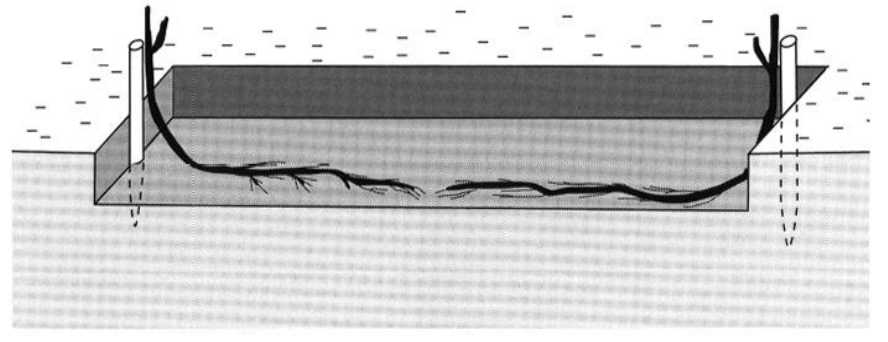

Fig. 10 - Restitution d'une fosse de plantation de type alveus avec deux plants à chaque extrémité (Pline, H. N., XVII, 35).

Des dispositions identiques ont été retrouvées aux Bosques (Ventabren), à Vernègues, à Alleins, Nîmes et Lattes. Toutes ces fosses allongées pourraient correspondre aux alvei décrits par Pline (H. N., XVII, 35) dans lesquels on dispose un plant à chaque extrémité, les racines se développant d'abord dans la partie médiane (fig. 10). À Château-Blanc (Ventabren), les fosses sont plus larges $(0,60 \mathrm{~m})$ mais correspondent au même dispositif. Autour des fosses et à la base de l'horizon travaillé, on trouve parfois des indices de labours croisés préalables à la plantation: le cas est particulièrement bien lisible dans le champ $l$ de La Quintarié (Boissinot, à paraître, fig. 9).

Les fosses cubiques de 3 pieds de côté préconisées par Pline (H. N., XVII, 35) ne figurent pas dans notre corpus parce que les rares exemplaires de ce type retrouvés en Gaule correspondent plutôt à des plantations d'arbres. Elles ne se présentent en effet jamais en série, à moins qu'elles ne soient disposées à grande distance les unes des autres ; dans ce cas, on les associe plutôt à des plantations d'arbres de type olivier, comme par exemple sur le site de La Ramière à Roquemaure (Pomarèdes, 1997). Les fosses isolées de ce genre, associées aux creusements déjà décrits, correspondent probablement à des arbres plantés au milieu des ceps.

Il est encore trop tôt pour se livrer à une typochronologie des traces de plantation. Il semble cependant que les vignobles les plus anciens (période hellénistique) présentent déjà une grande variété de dispositifs: tranchées, fosses plus ou moins ramassées (fig. 11). La chronologie observée sur l'un des sites les mieux datés, Le Florian à Nîmes, ne doit pas forcément être généralisée à l'ensemble de la région (Monteil $e t a l$., 1999) : les fosses allongées de la dernière phase de ce sitc sont aussi présentes à Lattes ou à Saint-Jean-du-Désert avant la conquête romaine. 


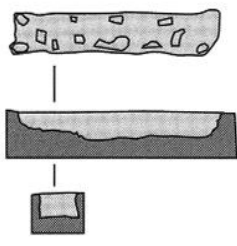

1
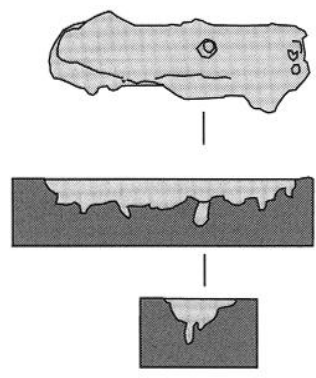

3

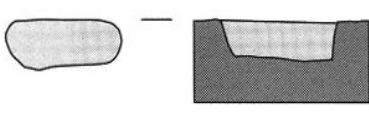

5

0 $1 \mathrm{~m}$

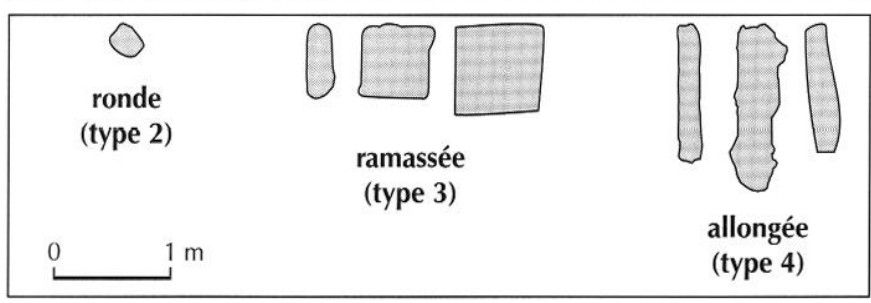

Fig. 11 - Essai de typologie des fosses de plantation: a, types $2 \grave{a} 4$ (le type 1 , non représenté, est constitué par les tranchées); b, plan et coupes de plusieurs fosses rencontrées; 1, 2, La Quintarié, champ b ; 3, 4, Derrière Saint-Pierre ; 5, Saint-Jean-du-Désert, champ b.

\section{CONDUITE DE LA VIGNE}

Durant l'Antiquité, plusieurs systèmes de conduite de la vigne étaient mis en œuvre dans les différentes provinces de l'Empire : les vignes rampantes, les vignes basses sans échalas, les vignes échalassées sans joug, les vignes à joug simple, les vignes à joug à quatre faces (jugum compluviatum) et les vignes sur arbre ou hautains (Billiard, 1913, p. 356-368). À la lecture des Agronomes, la première et la dernière méthodes sont clairement attestées en Gaule ${ }^{108}$. En est-il de même pour les données archéologiques disponibles pour l'instant?

En ce qui concerne la plantation de la vigne arbustive, des données morphologiques précises sont fournies par Columelle dans son traité sur Les arbres (Columelle, Les arbres, XVI, 2 et 3): les arbres porteurs sont d'abord installés dans des fosses carrées de 4 pieds de côté, ellesmêmes espacées de 40 pieds; après la pose de ces tuteurs, une nouvelle fosse (longueur : 4 pieds ; largeur : 2,5 pieds) est creusée à 1 pied du tronc pour y recevoir le plant de vigne provenant d'une pépinière. On ne connaît pas pour l'instant une telle configuration de fosses (espacements, emboîtements) dans les décapages archćologiques effectués dans le Midi. Mais la disposition peut être plus complexe encore si l'on suit les recommandations de Pline dans son Histoire naturelle (XVII, $35)$ : en effet, un arbre peut soutenir jusqu'à 10 ceps de vigne, ceux-ci étant plantés dans des fosses de 3 pieds de côté, en laissant un pied entre eux et l'arbre, selon une configuration que l'auteur ne précise guère. Quoi qu'il en soit, on imagine dans ce cas une profusion de fosses autour d'un réseau régulier de creusements plus volumineux. Là encore, aucun décapage archéologique ne permet d'illustrer cette technique.

Pour les vignes rampantes, procédé adapté aux régions balayées par des vents violents telle la Narbonnaise, deux techniques spécifiques peuvent être mises en évidence par l'archéologie. Elles sont toutes deux mises en œuvre dans l'espace intermédiaire laissé entre les rangées (planches) et concernent soit le renouvellement du cep (vigne couchée après avoir été taillée), soit l'entretien du terrain ; Columelle insiste particulièrement sur cette dernière technique que l'on peut trouver curieuse : « [...] mais après la taille, qu'il faut indispensablement faire en automne à ces sortes de vignes, on les renverse tout entières sur un intervalle d'entre les rangées différent de celui où elles étaient couchées auparavant, afin que la partie du terrain qu'elles avaient précédemment occupée puisse être fouillée ou labourée, et qu'après qu'on lui aura donné ces façons, on puisse les y remettre, et cultiver de même l'autre partie."

108. Pour la conduite ultra-basse de la vigne en Narbonnaise où souffle le violent mistral, voir Pline ( $H . N$., XIV, 3, 4), Columelle (De l'agriculture, V, 5) et Palladius (Traité d'agriculture, III, 14). En ce qui concerne la culture sur arbres, voir Columellc (IDe l'agriculture, $\mathrm{V}, 7$ ) et probablement Pline (H. N., XIV, 3, 2). 
(Columelle, De l'agriculture, V, 5, trad. Nisard éd., 1851, p. 291). De telles fosses transversales sont fréquemment repérées dans les décapages mais peuvent également relever de la technique du provignage.

L'ensemble des cas jusqu'à maintenant répertoriés ne permet pas d'identifier avec certitude une conduite basse de la vigne. En ce qui concerne l'utilisation des piquets, que ce soit des échalas, des jougs ou des éléments d'une architecture plus complexe, seules quelques découvertes isolées peuvent être mentionnées : au Florian, plusieurs fosses de calage de poteau ont été mises en évidence; leur diamètre ne dépasse pas $0,20 \mathrm{~m}$ et leur nombre semble inférieur aux fosses de plantation, trait caractéristique que les fouilleurs attribuent à une conduite haute sur jougs (Monteil et al., 1999). Dans la plupart des sites, la nature du terrain encaissant et la rapidité avec laquelle ce type de fouille est généralement mené ne permettent pas des observations aussi fines qu'au Florian et surtout qu'à Pompéi, où les configurations sont bien lisibles et les sections des échalas mesurables (Jashemski, 1996, p. 89).

\section{ORGANISATION DU VIGNOBLE}

Quel que soit le type de conduite, lorsqu'il est possible d'observer des séries de fosses ou de tranchées sur une grande surface, on ne distingue jamais en fouille de hiérarchie entre les différentes rangées qui sont quasiment toutes équidistantes et relativement étroites, sans aucune voie de desserte permettant l'aération du vignoble ou un éventuel classement des cépages. Contrairement à ce que l'on constate dans certains jardins de Pompéi, il n'y a pas pour l'instant dans le Midi de la Gaule de grand découpage autour d'un cardo et d'un decumanus ; si l'on en croit les Agronomes, cette disposition n'est pourtant pas caractéristique d'un microcosme tel que le jardin urbain ${ }^{109}$. En outre, l'organisation générale des vignobles fouillés en Narbonnaise interdit, comme nous l'avons montré, d'y placer des cultures intercalaires ainsi que l'usage des attelages pour les labours d'entretien.

Plusieurs dispositions sont présentes dans les vignobles antiques du Midi. La plantation en lignes se caractérise par des souches très rapprochées dans des

109. Voir Billiard (1913, p. 291) et les références antiques (Columelle, De l'agriculture, IV, 18 ; Pline, $H$. N., XVII, 35). rangées qui montrent des écartements plus grands. Le troisième état de la plantation du Florian à Nîmes (Monteil et al., 1999) peut se classer dans cette catégorie dans la mesure où les deux plants mis en place dans une même fosse sont plus rapprochés $(0,70-0,80 \mathrm{~m})$ que les rangées entre elles $(0,88 \mathrm{~m}$ en moyenne). Le champ $e$ de Saint-Jean-du-Désert relève du même principe puisque les ceps d'une même rangée sont distants de $1,25 \mathrm{~m}$ en moyenne pour un espacement de rangées variant entre $1,80 \mathrm{~m}$ et $1,90 \mathrm{~m}$ (Boissinot, Sauvage, 2000). La plantation en carré s'obtient en divisant le terrain en carrés ayant pour côtés l'espacement que l'on veut donner aux ceps (Chauzit, Chapelle, 1902, p. 145). À quelques degrés près (grille non orthogonale), le champ $a$ des Girardes se présente ainsi, avec un espacement de 5 pieds. Enfin, la plantation en quinconce se caractérise par un décalage entre les ceps d'une rangée à l'autre, de manière à ce que chaque plan occupe le sommet d'un triangle équilatéral. Les fosses ramassées de la deuxième phase du Florian correspondent à cette disposition avec des espacements proches de 3 pieds $(0,85 \mathrm{~m}$ en moyenne).

\section{FERTILISATION}

L'emploi de la fumure est évident sur la plupart des sites : de nombreux tessons érodés ou « frais ", répandus dans les fosses ou dans les horizons immédiatement susjacents, sont de bons indices de cette pratique de la fertilisation, antérieure ou postérieure à la mise en place des plants. Au Florian, des observations très précises ont été faites sur le remplissage des fosses de plantation (Monteil et al., 1999) : comme on peut le lire chez les Agronomes, l'apport d'engrais a eu lieu au moment du comblement des fosses (Billiard, 1913, p. 295). D'aspect "frais ", le mobilier archéologique correspondant à la partie imputrescible du fumier se compose de tessons aux arêtes vives, de charbons de bois et d'esquilles osseuses parfois brûlées; les pierres les plus grossières sont supposées assurer le maintien des plants dans la fosse.

\section{LE RENOUVELLEMENT DES PLANTATIONS}

Dans les vignobles méridionaux du XIX ${ }^{e} s$., il n'était pas rare de consacrer les deux tiers des frais de renouvellement des ceps à la technique du provignage, l'autre 


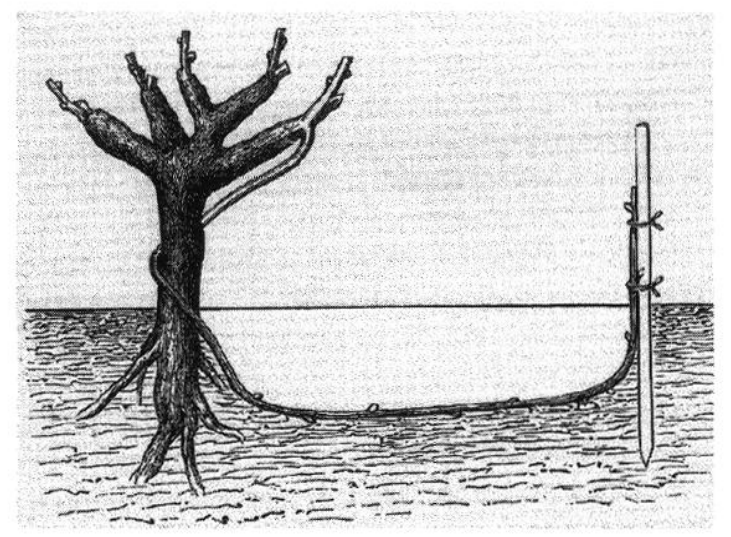

Fig. 12 - La technique du courbage (provignage d'un sarment dans une fosse contiguë. au cep) illustrée par un traité d'agriculture du débul du XX's. (Chauzit, Chapelle, 1902).

tiers relevant de la greffe (Villeneuve, 1829, IV, p. 469). $\mathrm{Si}$ le provignage ne concerne que le centième de la vigne planté, il est considéré comme le meilleur moyen de remplacement (Pellicot, 1866, p. 222), ne serait-ce que parce que le provin, à la différence des crocettes et des plans enracinés, donne du raisin l'annéc même où il a été couché (Rozier, 1772, p. 201). Il se pratique généralement de deux manières.

- Le courbage: recommandé plutôt pour les terrains humides, il consiste en l'enfouissement d'un sarment dans une fosse contiguë que l'on sépare ensuite du pied mère lorsqu'il est enraciné (fig. 12).

- Le cabus : spécifique du Midi, il s'opère en couchant dans une fosse une vigne pourvue de deux tiges que l'on fait ressortir en deux points, là où la vigne était initialement placéc et là où l'on souhaite disposcr d'un nouveau cep. On peut quelquefois remplir trois places avec la même souche, ce qui nécessite le creusement de fosses allongées suivant des directions variées (Pellicot, 1866, p. 222).

Le provignage par couchage d'une souche que l'on a refendue peut être considéré comme une variante du cabus, avec le danger accru d'un pourrissement du ccp trop exposé.

Il existe une quatrième méthode, moins pratiquée dans le sud de la France que les précédentes, qui consiste à utiliser un panier rempli de terre pour enraciner un sarment à proximité de la souche mère (Bidet, 1752, p. 11-12; Rozier, 1772, p. 177). Ce procédé facilite le transport du nouveau plant qui pourra éventuellement être replanté dans une nouvelle rangée.
Toutes les pratiques viticoles méridionales dont nous venons de parler se placent dans la tradition antique et il n'y a guère d'innovation en ce domaine. Nous avons préféré adopter les dénominations modernes parce qu'elles sont plus précises que le terme latin de propagatio, qui englobe l'ensemble des pratiques (Billiard, 1913, p. 271-277) ; en outre, les textes des modernes ou contemporains sont en général plus détaillés que ceux des Agronomes de l'Antiquité et éclairent certaines " incohérences" dans la composition des ouvrages les plus anciens.

Comment peut-on reconnaître sur le terrain la pratique de tels procédés de renouvellement des ceps ? Si le réseau de fosses ou de fossés mis en évidence se présente seul, sans aucun autre ensemble dc crcuscments, il n'y a aucune chance de mettre en évidence quoi que ce soit; si provignage il y a eu, celui-ci a laissé des traces trop superficielles pour être enregistrées dans le sous-sol. Lorsque l'on distingue plusieurs réseaux aux morphologies différentes, la première question concerne leur éventuelle corrélation : s'agit-il d'une simple superposition de traces correspondant à deux mises en culture indépendantes, ou bien, existe-t-il un lien de dépendance entre les emplacements des éléments de chaque réseau ? L'analyse statistique entreprise sur les fosses décapées aux Girardes montre que les creusements les plus allongés ne sont pas situés n'importe où par rapport aux fosses rectangulaires initiales (fig. 13): l'axe des rangées a été surtout choisi ( $92 \%$ des cas) préférentiellement de façon à être tangent aux fosses. De toute évidence, il s'agit là soit de manières culturales associées à l'entretien du vignoble (désherbage, déchaussage), soit de la technique du provignage. La base des creusements linéaires étant au même niveau que celle des fosses et leur présence n'obéissant à aucune périodicité, il est préférable de retenir la seconde hypothèse. Dans ce cas, on en déduit que le provignage ne s'est effectué que dans l'axe des rangées.

Il existe de nombrcux cas où les deux séries présentent des directions perpendiculaires. Nous avons déjà vu qu'il pouvait s'agir d'une technique d'entretien par basculement des souches. L'absence de périodicité dans ce type de creusement nous pousse cependant à privilégier l'hypothèse d'un provignage occasionnel. Dans le cas des fosses allongées de type alvei, il n'est guère étonnant que les recreusements perpendiculaires occupent quasi exclusivement l'extrémité des segments. 


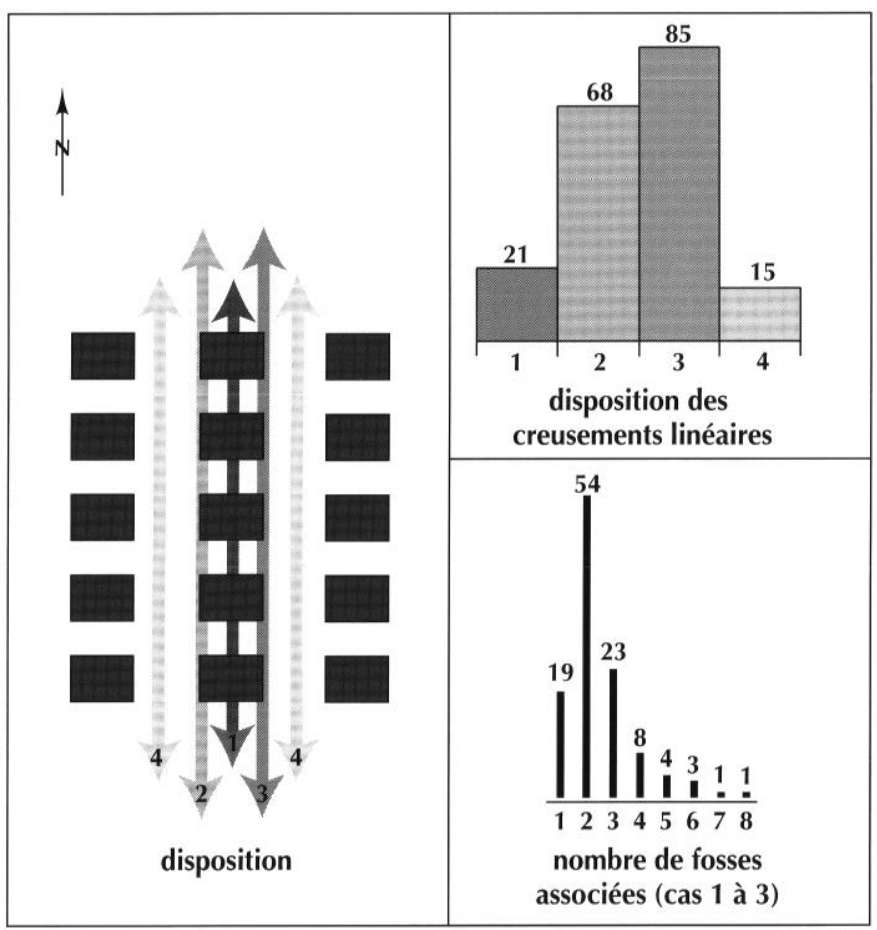

Fig. 13 - Disposition des creusements linéaires (l à 4 ) par rapport aux fosses rectangulaires des vignobles des Girardes à Lapalud (Haut-Empire). À droite, décompte des dispositions observées et du nombre de fosses reliées par ces mêmes creusements. Les résultats montrent qu'il s'agit bien là de la technique du provignage.

Plus rarement, des creusements linéaires obliques se présentent en certains points. Il peut s'agir là de provignages multiples à partir d'une même souche. Ce phénomène s'observe très bien autour des fosses de bordure du champ $l$ de La Quintarié (fig. 9). L'absence de fouille systématique nous prive cependant d'une détermination précise de la technique (cabus ou courbage ?).

Lorsque l'on provigne, on peut directement remplacer le cep manquant à la place choisie. Ainsi, de provins en provins, on parvient à renouveler entièrement un vignoble.

Si l'on tient compte de l'ensemble des fosses de provignage visibles sur certains plans de fouille, le vignoble peut nous paraître particulièrement désordonné, perdant la disposition régulière de la première plantation. On ne s'étonne plus alors des remarques de O. de Serres (1605, p. 180-181): "Sans distinction d'ordre ni de rang, en provignant, asseoit-on les sarmens où mieux se rencontre, près ou loin les uns des autres : comme aussi un, deux ou trois ceps sortiront de chaque

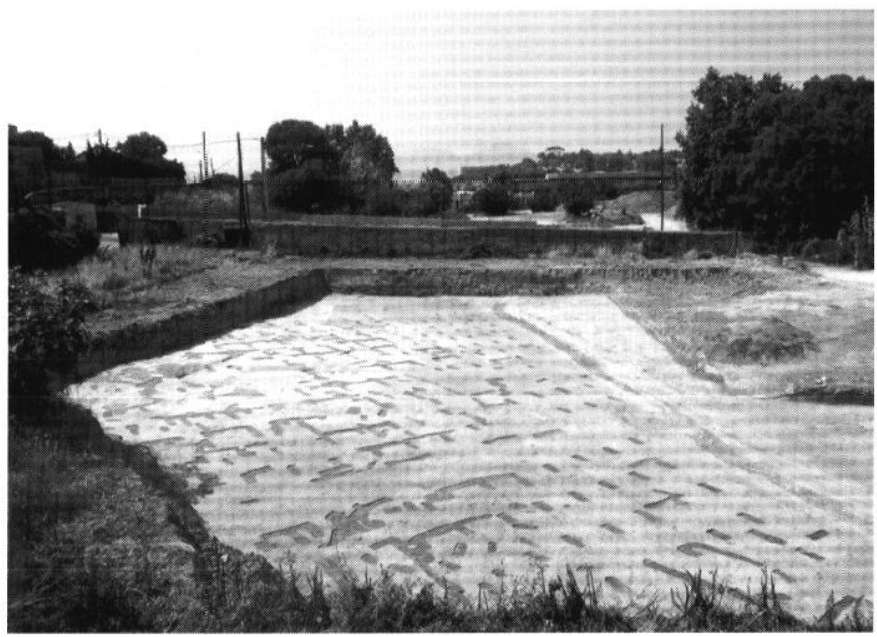

Fig. 14 - Vue vers le sud du champ e de Saint-Jean-du-Désert à Marseille (époque hellénistique). Le ruisseau fossile est à droite. Le vignoble montre de nombreux indices de la technique du provignage.

souche, ainsi que mieux s'accordera: car sans nulle direction de ligne ni de distance, ouvre t'on en cet endroit. Par telle méthode, la Vigne eschalassée perd son alignement et mesure, se confondant entièrement es rang et ordre, sous lequel aura été placée, que la basse conserve jusqu'à la fin. Aussi, de droite et large qu'elle était à son commencement, par le provigner se rend tordue el étroite, voire tellement pressée de ceps, que l'accez par entre eux étant toujours difficile et quelquefois nuisible, on n'y passe que par nécessité. » Si l'on tient compte de tous les creusements, le champ $e$ de Saint-Jeandu-Désert peut être appréhendé comme un ensemble désordonné à la suite du provignage, avec des rangées plus rapprochées et plus nombreuses (fig. 14 et 15). Une telle hypothèse n'est évidemment pas compatible avec l'idée d'un renouvellement systématique du vignoble. À partir des mêmes données, on peut toutefois supposer que les provins n'ont pas tous grossi à l'endroit où ils se sont initialement enracinés et qu'ils ont été replantés dans les parties manquantes du vignoble. Dans ce cas, une certaine régularité peut toujours être envisagée.

\section{LES VIGNOBLES DANS LES PAYSAGES AGRAIRES}

Il n'a pas été nécessaire d'attendre la découverte de traces agraires pour pouvoir aborder la question des productions viticoles dans les domaines antiques. Depuis 


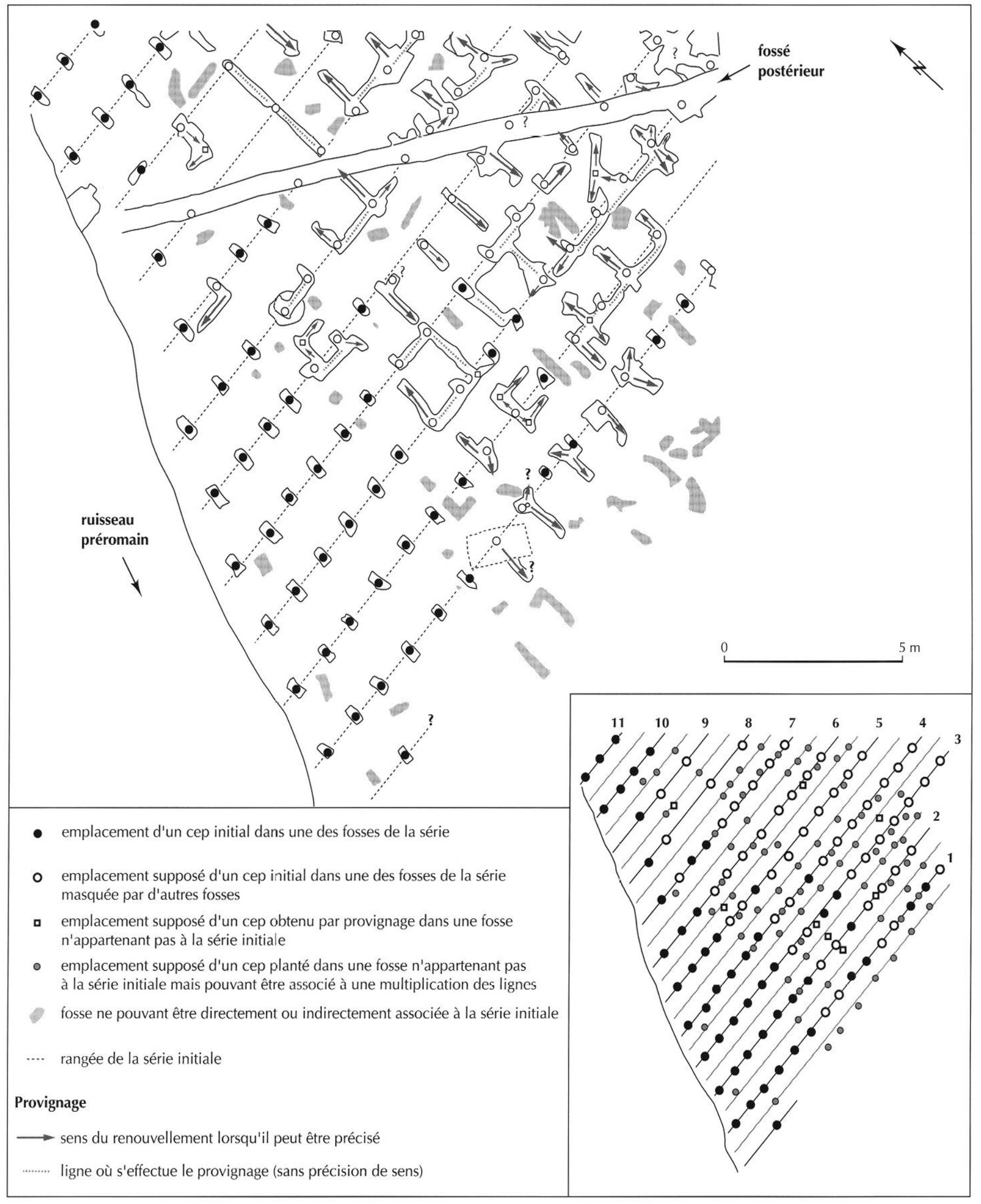

Fig. 15 - Plan des structures du champ e de Saint-Jean-du-Désert à Marseille (période hellénistique), avec essai de localisation des tranchées de provignage et, en bas à droite, hypothèse de restitution du vignoble dans le cas où le marcottage permet le doublement du nombre de rangées; dans ce cadre, toutes les fosses repérées à la fouille sonı mises en relation avec cette pratique supposée. Il s'agit là d'une hypothèse parmi d'autres. 


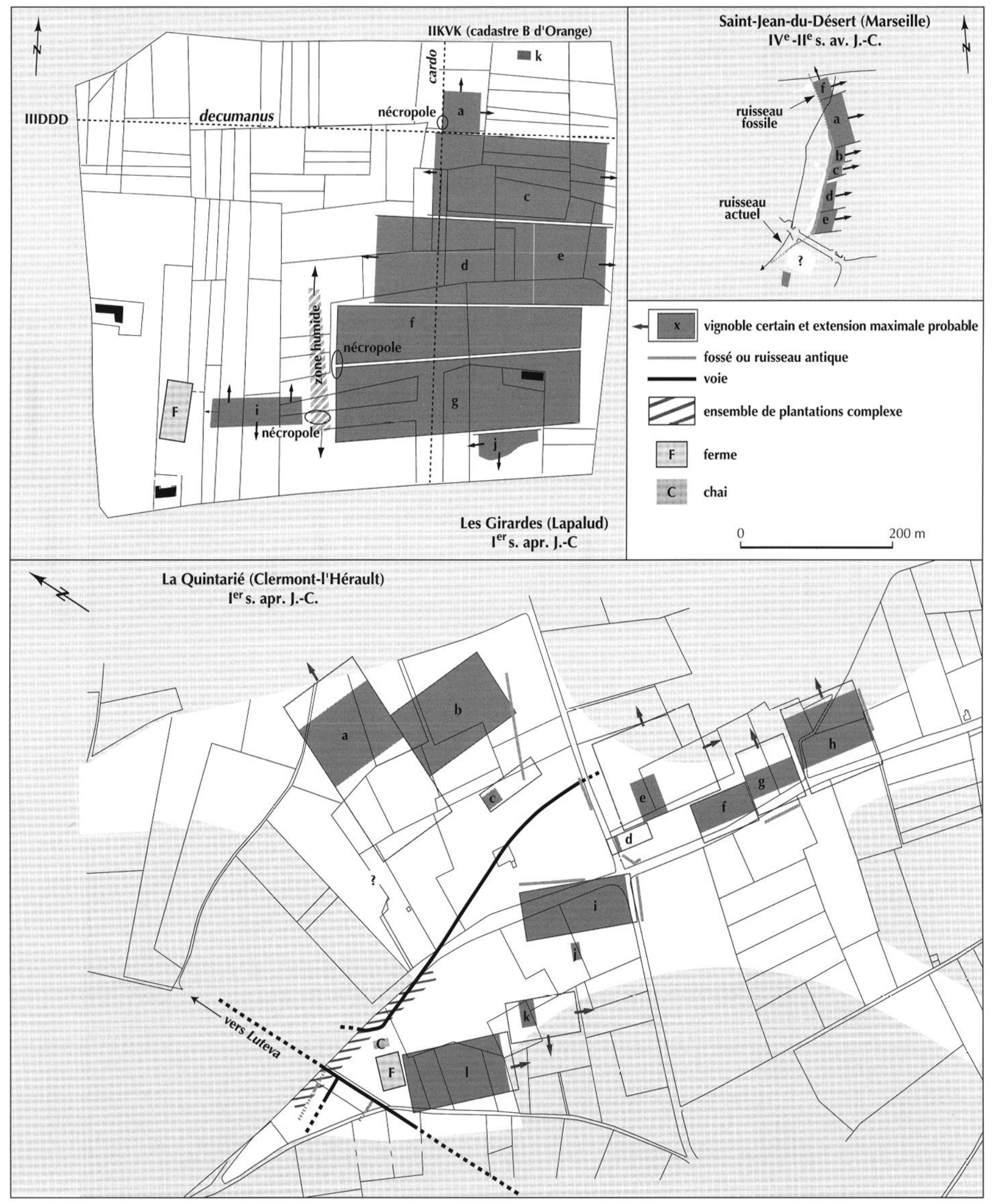

Fig. 16 - Plans de quelques terroirs viticoles mis en évidence par l'archéologie préventive (Les Girardes, Saint-Jean-du-Désert et La Quintarié). Les champs représentés ne constituent qu'une partie des surfaces présentant des traces agraires; nous avons privilégié les ensembles qui pouvaient être circonscrits (dénommés par une lettre en minuscule). 
longtemps déjà il est même possible d'évaluer l'ampleur des vignobles à partir de la capacité de stockage des chais associés aux aires de vinification (Tchernia, 1995b). Ainsi, on connaît dans la plaine du Tricastin un des plus grands domaines viticoles de la Gaule (Le Molard à Donzère) dont la superficie est certainement comprise entre 71 et 166,6 ha si l'on tient compte de la nature du terroir (Odiot, 1996, p. 19). Avec de telles dimensions, il y a peu de chances que l'archéologie agraire puisse un jour prétendre circonscrire l'ensemble d'un vignoble!

En dehors des découvertes exceptionnelles dans le territoire de Chersonèse de Taurique (Chtcheglov, 1992) ou dans les jardins de Pompéi (Jashemski, 1979, 1996), l'organisation et la disposition des vignobles n'étaient jusqu'à présent abordées qu'à partir de clichés aériens particulièrement révélateurs. En Italie, des résultats très intéressants ont été obtenus dans la région du Tavoliere (Bradford, 1950 ; Jones, 1980 ; Riley, 1992 ; Chouquer, Favory, 1991) : autour des fermes antiques et des chemins, on peut distinguer plusieurs procédés de plantation (fosses et fossés) et parfois même des traces de provignage.

Maintenant que la morphologie des plantations est bien connue, la fouille sur de vastes surfaces est en général mise en ouvre à l'occasion de grands travaux (échangeurs autoroutiers, zones d'emprunt de matériaux), dans le cadre de l'archéologie préventive. Trois exemples récents démontrent la contribution de l'étude des plantations au cadre plus général des paysages agraires de l'Antiquité (fig. 16).

\section{SAINT-JEAN-DU-DÉSERT À MARSEILLE}

Ce gisement, situé à environ $4 \mathrm{~km}$ à l'est du centre historique de Marseille et fouillé à partir de 1992, a livré de nombreuses traces attribuables à la viticulture dans un secteur considéré comme appartenant au terroir de la ville grecque de Marseille (Bats, 1986a), en dehors de tout contexte d'habitat et de cadastre ${ }^{110}$ (Boissinot, 1995b ; Boissinot, Sauvage, 2000). Les champs, probablc-

110. Aucunc étude morphologique n'a été à ce jour envisagéc sur l'ensemble du terroir très urbanisé du bassin de Marseille, comme ccla a pu être tenté aux environs de la colonie grecque d'Olbia à Hyères (Benoit, 1985). Les fouilles de Saint-Jean-du-Déscrt livrent certes plusieurs limites de parcelles, plus ou moins parallèles; elles ne peuvent cependant pas être intégrées pour l'instant dans un quadrillage de plus grande extension. ment disposés en lanières le long d'un petit ruisseau, montrent l'emploi d'au moins deux types de plantation dans le courant de la période hellénistique (IVe-II $\mathrm{e}$. avant J.-C.), datation obtenue à partir des recouvrements sédimentaires observés et du mobilier résiducl découvert en stratigraphie. On dénombre six parcelles dans la partie nord (fig. 17, $a$ à $f$ ), le secteur méridional étant plus difficile à lire à cause des perturbations occasionnées par l'installation d'un habitat de l'Antiquité tardive. La rive droite du vallon est occupée par d'autres plantations qui n'ont pas pu être entièrement dégagées et, en conséquence, organisées sous forme de parcelles. Tous les vignobles mis en évidence présentent des largeurs différentes. Le plus grand d'entre eux est constitué d'une série de 50 tranchées parallèles comportant des logettes de provignage (fig. 6 et fig. 17,a). Quelques rares plantations d'arbres sont associées aux vignes. Les autres champs montrent plutôt l'emploi des fosses disposées en rangées avec, localement, quelques indices de provignage (fig. 17, $d$ et $e$ ). Les limites entre les champs sont matérialisées par des fossés ou des barrières végétales ${ }^{111}$. L'ensemble des parcelles occupant un fond de vallon où les sources sont nombreuses, il n'est pas étonnant que des travaux de drainage importants aient été entrepris dès cette époque: dans le champ a, deux tranchées remplies de galets permettent d'évacuer les eux souterraines vers le ruisseau (maintenant) fossile (fig. 6 et Boissinot, 1995b, fig. $4, \mathrm{n}^{\text {os }} 2$ et 4 ) ; dans la partie méridionale du champ $d$, un fossé draine les eaux superficielles provenant des sources situées en amont.

I.e site est encore occupé durant la période galloromaine: les berges du ruisseau sont aménagées par des murettes et plusicurs fossés sont creusés, parfois à l'emplacement de divisions anciennes. Les traces agraires de cette période, malheureusement difficiles à lire dans des enscmbles homogènes, ne permettent pas de connaitre l'évolution des cultures avant qu'une crue importante ne vienne définitivement recouvrir l'ensemble des vestiges dans le courant du II ${ }^{\mathrm{e}} \mathrm{s}$. après J.-C.

111. Entre les champs $d$ ct $e$, le fossé de séparation montre en profondeur l'emplacement de plusicurs calages aménagés avec des galets, qui suggèrent la mise en place de plusicurs piquets. En outre, cette ligne est soulignée dans la partie haute du remplissage par un amoncellement remarquable de coquilles d'escargots terrestres visible dès le décapage. Les autres limites de parcelles observées sont matérialisées par des fossés. L'un deux est visible sur l'une des coupes publiées dans Boissinot, à paraître, lig. 5 . 


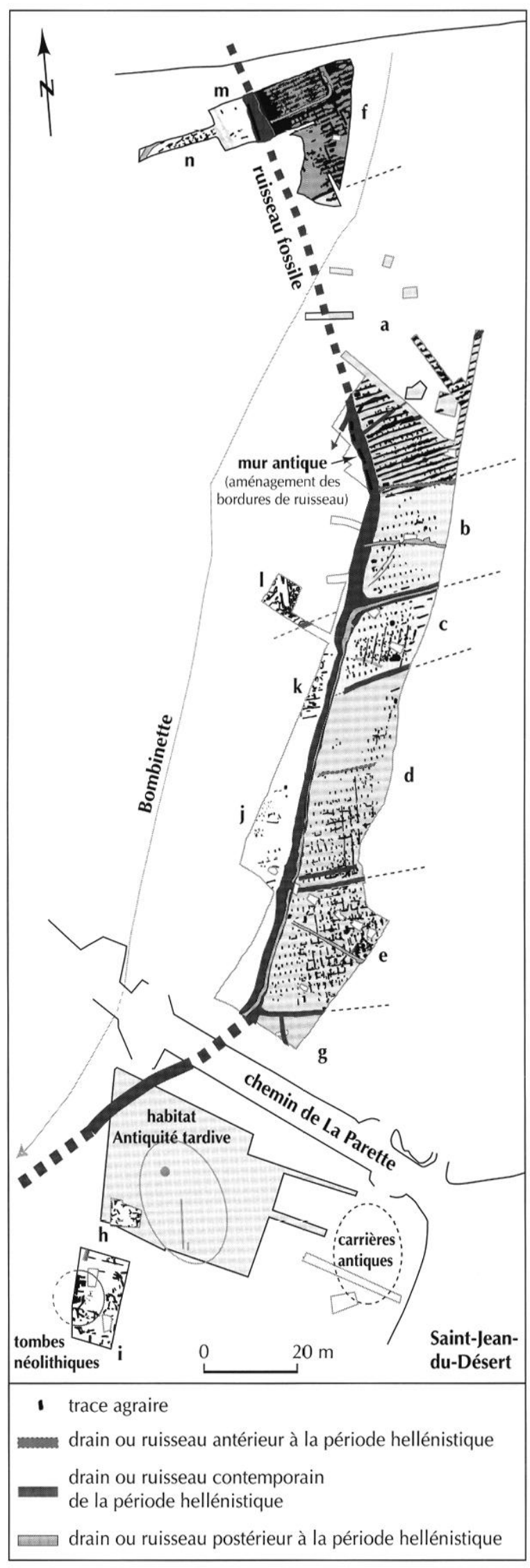

Fig. 17 - Plan du parcellaire de Saint-Jean-du-Désert à Marseille (toutes périodes confondues). Les champs circonscrits par la fouille sont dénommés par des lettres (a $a ̀ \mathrm{n})$.

\section{LES GIRARDES À LAPALUD}

Cet ensemble qui s'inscrit dans le cadastre B d'Orange au cœur de la plaine du Tricastin (Vaucluse) est de loin le plus vaste fouillé à ce jour. Les décapages et les sondages réalisés dans le cadre de l'opération TGV-Méditerranée permettent d'estimer à plus de 20 ha la surface dévolue à la viticulture autour d'une ferme comportant une nécropole et des chemins de desserte. Les deux centuries méridionales comportent plusieurs champs contigus constitués de petites fosses de plantation rectangulaires datés du Haut-Empire (fig. 16, 18 et 19). À ces trous de plantation sont associés des creusements linéaires que l'on peut interpréter comme des traces de provignage (voir supra). Seuls deux champs ont pu être entièrement circonscrits (fig. 16, $f: 330 \mathrm{~m} \times 75-45 \mathrm{~m}$ et $g: 330 \mathrm{~m}$ x $96 \mathrm{~m}$ ) ; leurs formes dénotent l'existence d'un changement d'orientation dans le parcellaire. Au nord de cet ensemble, deux champs ( $d$ et $c$ ) aux largeurs proches (96 et $108 \mathrm{~m}$ ) n'ont pu être entièrement délimités ; on notera qu'ils ne matérialisent en rien l'emplacement du cardo dans ce secteur: en effet, la projection au sol du cadastre B d'Orange restitué à partir des travaux de G. Chouquer ${ }^{112}$ ne correspond ici à aucune modification notable dans l'ordre des fosses ${ }^{113}$. D'une manière générale et à l'inverse des observations précédentes, les limites de vignobles sont soulignées par des fossés peu profonds, des chemins ou une simple interruption (momentanée) des traces de culture. D'autres découpages peuvent être effectués, notamment entre $d$ et $e$, où un léger décalage des fosses de plantation peut être observé ; il s'agit peut-être là d'une interruption provisoire de la mise en placc du vignoblc, sc poursuivant ensuite vers l'est ou l'ouest. La centurie située au nord-est comporte deux types de fosses différents: des carrés espacés de 5 pieds dans tous les sens, selon une trame qui

112. Voir, en dernier lieu, l'ouvrage général de G. Chouquer et F. Favory (1991), à partir des travaux pionniers de A. Piganiol (1962).

113. Même en considérant un intervalle d'incertitude important pour la projection au sol effectuée à l'aide du théodolite (travaux de Laisné et Tripeau). Ce découpage existe bien dans la partie nord puisqu'il correspond à la limite occidentale du champ $a$. 


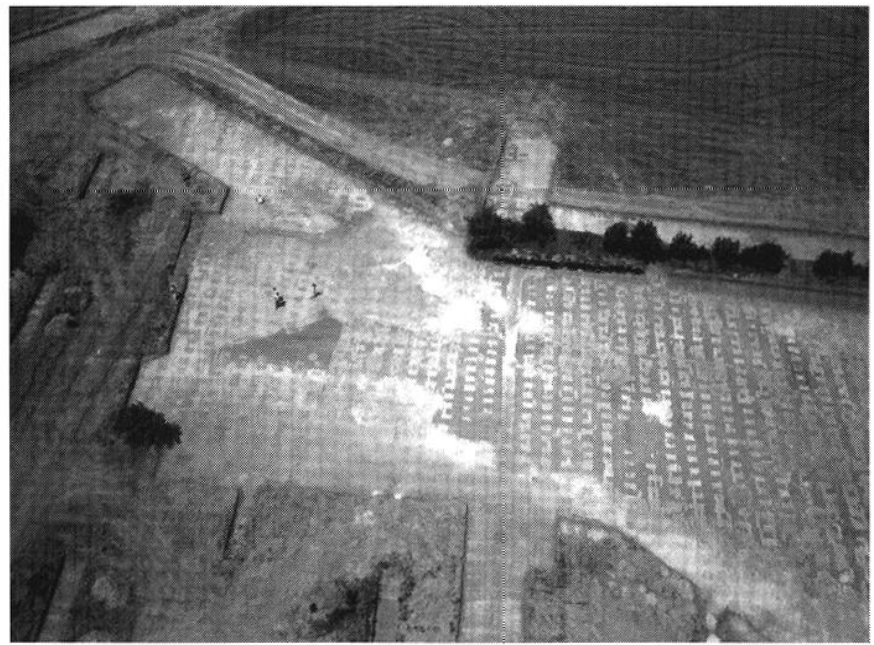

Fig. 18 - Vue vers le nord de la partie septentrionale du champ d des Girardes à Lapalud (Haut-Empire). La limite entre les vignobles $\mathrm{d}$ el $\mathrm{c}$ est encro marquée par une haie.

n'est nas orthogonale (fig. 16, $a$ et fig. 20), et des petites fosses circulaires espacées de 4 pieds suivant toutes les directions (fig. 16, $k$ ). L'examen des recouvrements et du mobilier découvert dans le remplissage des structures agraires permet de dater ce premier ensemble du $\mathrm{I}^{\mathrm{cr}}$ s. de notre ère. Dans le secteur du croisement entre cardo et decumanus, les fosses du vignoble sont creusées aux dépens d'une nécropole à incinération attribuée au milieu de ce même siècle.

Dans un deuxième temps et sur toute la partie orientale du terrain, plusieurs fossés de drainage sont mis en place dans des parcelles qui ne sont peut-être plus entièrement dévolues à la viticulture.

\section{LA QUINTARIÉ À CLERMONT-L'HÉRAULT}

À l'inverse des deux sites précédents, les champs mis en évidence (fig. 16, $a$ à $l$ ne forment pas un ensemble compact. Les vignobles repérés semblent en effet dispersés dans le terroir suivant des orientations variées. Un seul champ a pu être entièrement circonscrit: le champ $b$ mesure $137,1 \mathrm{~m}$ de long pour une largeur de $89,3 \mathrm{~m}$, soit une surface à peine supérieure à 1,2 ha (fig. 16). D'autres parcelles sont beaucoup moins étendues et, bien qu'incomplètement explorées, ne paraissent pas dépasser les 0,4 ha (champ $f$ ). Nous ignorons pour l'instant quels sont les liens que ces champs entretiennent avec les éventuels cadastres de la

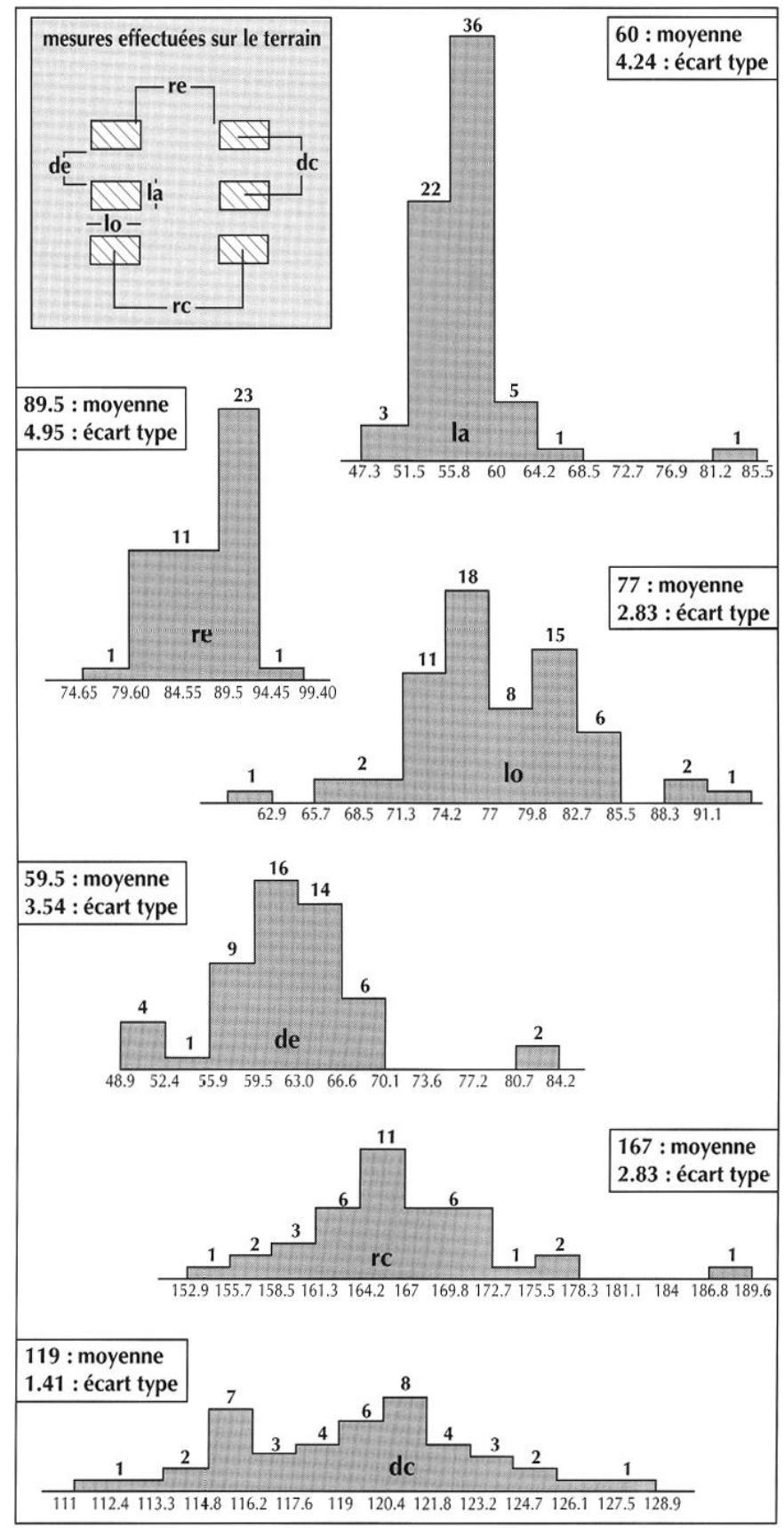

Fig. 19 - Histogrammes des principales mesures effectuées sur les fosses de plantation du champ $\mathrm{f}$ des Cirardes à Lapalud (HautEmpire).

terrasse alluviale de la vallée de l'Hérault (Pere», 1995). Si l'on considère que tous les champs sont contemporains, il faut peut-être admettre que le paysage agraire comportait déjà une grande variété de formes avant que le vignoble ne soit établi. Les éléments de datation recueillis à proximité de la ferme nous permettent de dater cet ensemble du ${ }^{\text {er }}$ s. après J.-C. ; à cet endroit, le 


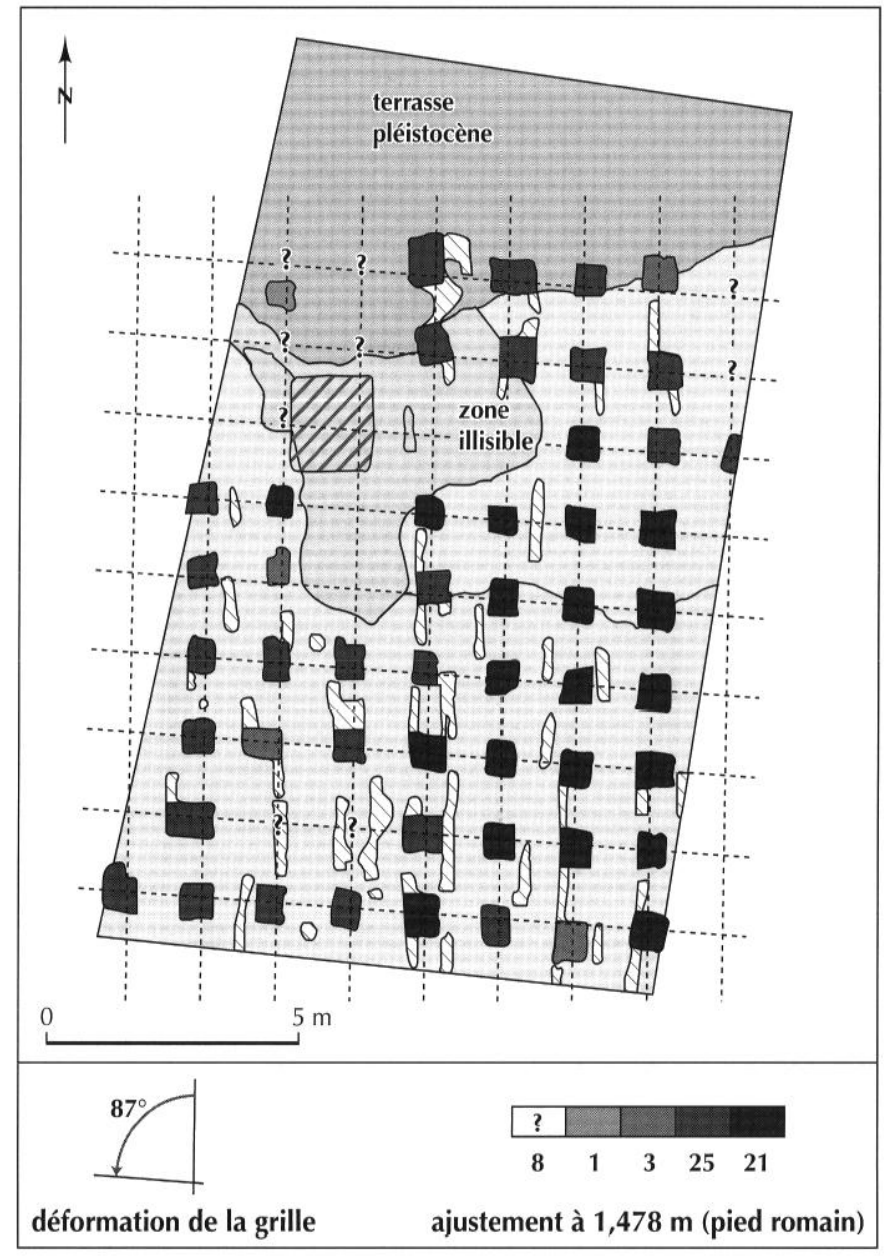

Fig. 20 - Relevé de terrain des fosses de plantation du champ a des Girardes à Lapalud (Haut-Empire) avec superposition d'une grille non orthogonale de 5 pieds romains de côté. La qualité de l'ajustement est indiquée en haut à droite, le gris est d'autant plus sombre que la coïncidence est meilleure (dessin : G. Alfonso).

champ $l$ suit le mur de façade du bâtiment du HautEmpire, avant d'être recouvert par des remblais de l'Antiquité tardive. Toutes les plantations sont constituées de fosses allongées que l'on peut interpréter comme des alvei (fig. 9). La réalisation du provignage n'est pas systématique et un seul champ, ultérieurement recouvert par un chemin, montre la mise en place de deux plantations successives. Il est intéressant de noter que la ferme comporte un chai à dolia ${ }^{114}$, quatre cuves et un pressoir dans sa partie septentrionale, démontrant que la vinification s'effectuait bien sur place, dans un habitat qui fut aussi un relais sur la voie reliant Cessero à Luteva (Pomarèdes, 1999, p. 129).

114. Fouille et aimable renseignement de $\mathrm{H}$. Pomarèdes.

\section{CONCLUSIONS ET PISTES DE RECHERCHE}

Après presque une décennie d'une pratique « indisciplinée » de l'archéologie agraire, il est désormais possible d'appréhender l'organisation des terroirs antiques sur de vastes surfaces, surtout lorsque des plantations arboricoles viennent s'inscrire profondément dans le sous-sol. Même si quelques points d'interrogation demeurent, on reconnaît maintenant sans trop d'hésitations les traces de la viticulture, que l'on ne confond plus, par exemple, avec des négatifs de solives en bois pour supporter une habitation ou un pont.

Une méthode qui a fait ses preuves permet de livrer un contexte de production et de dater des vestiges forcément pauvres en indices chronologiques; il suffit de partir de l'habitat et de ses éventuelles structures de vinification pour gagner ensuite les champs mis en place dans un réseau de chemins et de fossés (Boissinot, Brochier, 1997, p. 52-53). On trouve généralement aux abords des sites suffisamment de matériaux et de relations stratigraphiques pour assurer une datation; avec les réseaux associés, il est possible de retrouver une cohérence morphologique au-delà du point de départ et d'envisager l'étendue d'un terroir.

Le corpus des traces de plantation est désormais bien fourni, sans être complet pour autant. Là, comme dans d'autres domaines de la production, les possibilités d'invention sont plus grandes que ne le laissent croire les traités techniques. En additionnant les procédés vantés aux " erreurs " livrées par les Agronomes à titre de contre-exemple, la liste des cas s'allonge déjà très rapidement. Tout travail typologique élaboré à partir du corpus de terrain devra cependant veiller à bien séparer ce qui relève de la plantation proprement dite de ce qui peut être attribué au renouvellement ou à la genèse du vignoble, sources de très nombreuses variations. Plusieurs cas évoqués plus haut montrent que cette distinction n'est pas toujours aisée ${ }^{115}$.

Le faible nombre de cas analysés jusqu'à présent en Gaule et le retard de ce type de recherche en Grèce ou en Italie ne permettent pas de reconnaître pour l'instant des

115. Cependant, lorsque les observations de terrain se multiplient, l'audace interprétative se fait plus grande, la répétition des pratiques étant plus probable que l'invention continuelle. Ainsi, certaines formes complexes que nous n'osions interpréter dès les premières découvertes ont-elles pu se ramener à la superposition de cas plus simples observés ailleurs. 
façons propres à chacune de ces régions. Dans ce domaine comparatif, nos seules informations proviennent de la lecture des Agronomes. On peut toutefois imaginer dans l'avenir pouvoir suspecter la présence de nouveaux colons grâce à l'introduction de façons culturales inconnues dans la région où ils viennent s'implanter. $\mathrm{Si}$, par exemple, les fosses rectangulaires retrouvées en nombre aux Girardes ne s'avèrent être présentes, comme c'est le cas actuellement, que dans le contexte du cadastre B d'Orange, il faudra s'interroger sur la tradition agronomique du pays d'origine des colons de la $\mathrm{II}^{\mathrm{e}}$ légion Gallica.

Dans le cadre de l'inventaire des formes de plantation, plusieurs vérifications sont nécessaires : a-t-on réellement pratiqué la culture arbustive de la vigne en Gaule Narbonnaise? Les constructions complexes à l'aide de jougs ont-elles été utilisées en dehors des jardins, dans le cadre de l'arboriculture extensive ?

En ce qui concerne la chaîne opératoire, des analyses plus fines que celles conduites généralement permettraient d'en savoir plus sur la nature du défrichement préalable, les outils utilisés pour le creusement, la gestuelle et les procédés de rebouchage. Sur plusieurs sites explorés au cours de l'opération TGV-Méditerranée, une croûte calcaire, postérieure au creusement des fosses, souligne la limite du volume de terre remué par les agriculteurs et révèle en de nombreux points les impacts ou déplacements des outils agricoles et l'empreinte de quelques racines. Avec un décapage fin à l'aspirateur, des relevés précis de type photogrammétrique et des expérimentations conduites en parallèle sur le même sédiment, on peut espérer retrouver la forme des outils, leur utilisation et la nature physique du terrain au moment du creusement. En suivant une approche pluridisciplinaire (malacologie, sédimentologie, micromorphologie, anthracologie et palynologie), il est possible d'envisager avec précision les techniques de comblement des fosses et une partie des soins culturaux apportés au vignoble, ceux inscrits dans la partie la plus basse du remplissage restée en dehors des perturbations agraires postérieures (Boissinot, 1997). Pour suivre ce progamme, il faut désormais considérer la trace agraire comme un objet de recherche à part entière et lui accorder, au moins de manière partielle, autant d'attention qu'on le ferait pour une structure plus « prestigieuse » (sépulture, habitat) ${ }^{116}$.

116. Dans le cadre de la programmation de la recherche archéologique, un tel travail pourrait s'insérer dans les progammes 20
D'un point de vue morphologique, les champs de plantation constituent des réseaux de fosses (ou de fossés) régulièrement réparties, creusées d'un seul jet puis aussitôt rebouchées. Pour ces raisons, elles constituent des pièges à sédiment et des repères précieux pour une restitution de la topographie ancienne. Ainsi, en comparant le pendage des lignes reliant la base de toutes les fosses avec celui des interfaces des niveaux les surmontant, y compris celle entre le dernier sol cultivé et l'atmosphère, il est possible de proposer un bilan des phénomènes d'érosion et d'accrétion qui ont affecté la parcelle dans la diachronie. En outre, le remplissage des fosses nous informe sur l'existence d'anciens sols ultérieurement décapés, conservés en position secondaire dans les fosses. En spatialisant les informations, il devient possible de restituer l'ancien paysage précédant la mise en place du vignoble (Boissinot, à paraitre). Dans ce cadre géoarchéologique, il est possible de suspecter un éventuel tri préalable du sédiment encaissant (épierrement par exemple) ou l'apport massif d'amendements visant à améliorer le soussol du vignoble.

Nous savons d'après les Agronomes que l'implantation des fosses s'effectuait soigneusement à l'aide de cordeaux et de jalons, en respectant dans la plupart des cas des rapports entiers entre les différents espacements. Si l'on part de cette hypothèse, il est possible de s'interroger sur l'utilisation effective du pied romain de 0,2957 m dans l'ensemble des vignobles de l'époque romaine. En mesurant précisément l'ensemble des espacements et en superposant aux relevés des grilles dont les cotes sont des multiples de cette unité de mesure, on peut tester l'universalité de son emploi ou repérer d'éventuels systèmes locaux. Ainsi, à La Quintarié, la recherche d'un rapport entier entre les espacements amène plutôt la reconnaissance d'un pied proche de $0,22 \mathrm{~m}$.

Sur le plan historique, plusieurs questions peuvent être discutées à partir de la découverte de vestiges de vignobles. En premier lieu, celles qui concernent la diffusion de la viticulture à partir d'une colonie installée dans une région

\footnotetext{
« Espace rural, peuplement et productions agricoles aux époques galloromaine, médiévale et moderne » et 31 "Anthropisation ct amćnagement des milicux durant l'Holocène ". La fouille de traces agraires en dehors de tout contexte d'urgence permettrait de définir un premier protocole d'observation.
} 
où cette technique est ignorée. On pense évidemment au cas de Marseille grecque et de son environnement indigène. Après la diffusion du vin étrusque, la métropole grecque crée au milieu du $\mathrm{VI}^{\mathrm{e}} \mathrm{s}$. avant J.-C. ses propres vignobles, comme en témoignent les premières productions d'amphores locales (Bats dir., 1990). À $4 \mathrm{~km} \mathrm{du}$ centre historique, les découvertes de Saint-Jean-du-Désert donnent une idée de la progression de la viticulture quelques siècles après, dans un secteur que l'on suppose appartenir à la chôra. Il ne s'agit là que d'une extension minimale et on ignore si les mains indigènes n'ont pas participé au creusement des plantations. En dehors de ce territoire et sur la rive gauche du Rhône, on ne possède pour l'instant que quelques macrorestes végétaux (pépins carbonisés) qui, lorsqu'ils ne peuvent être interprétés comme des résidus de moût, restent des témoignages évidents de la consommation du raisin et non de la pratique effective de la viticulture. On songe alors à l'intérêt de fouilles conduites dans les champs à proximité des sites ayant livré de tels macrorestes.

Pour les périodes plus récentes, d'autres questions peuvent être envisagées, comme celle de l'application de la décision du Sénat romain visant à interdire la plantation de la vigne et de l'olivier en Gaule transalpine à la fin du II $^{\mathrm{e}}$ s. avant J.-C. (Cicéron, De Republica, III, 9, 16). La découverte de vignobles datés de cette période en milieu indigène pose évidemment le problème du degré et de la durée d'application d'un tel décret connu par cette seule source. Une telle discussion peut être envisagée à partir des découvertes du Florian à Nîmes (Monteil et al., 1999), maintenant que l'on sait d'après les analyses de A. Aymard (1948) que la phrase de Cicéron se traduit plutôt par: " [...] donner plus de prix aux oliveraies et aux vignobles appartenant à nos ressortissants ", à savoir les citoyens romains de plein droit, ou les colons jouissant du droit latin, comme le précise R. Dion (1959, p. 99), précision qui engage à s'intéresser de plus près au statut du propriétaire, dictant à lui seul l'application dudit décret et la mise en place éventuelle d'un vignoble à cette période.

$\mathrm{La}$ recherche de vestiges de vignoble antiques pourra encore s'appliquer à des questions économiques, comme, par exemple, celle de l'éventuelle proximité entre ateliers d'amphores (conteneurs) et plantations (contenus). En suivant cette approche, il devient maintenant possible de tester les intuitions de R. Dion (ibid.) sur la possible ancienneté du vignoble en pays rutène (Gaillac) ou allobroge (Côte-Rôtie et Hermitage).

Enfin, puisque oliviers et vignes sont souvent cultivés conjointement en milieu méditerranéen, selon des façons culturales faciles à distinguer, il n'est plus désormais impossible de définir la part de l'un et de l'autre dans des établissements où l'incertitude sur la production demeure, les critères de reconnaissance des installations oléicoles et vinicoles n'ayant pas un caractère absolu (Brun, 1993a).

La fouille de vignobles constitue un élément parmi d'autres permettant d'enrichir notre questionnaire sur la viticulture antique (mise en place, fonctionnement, abandon). Palliant parfois les lacunes de la connaissance ou l'ambiguïté des informations, cette approche s'intègre particulièrement bien aux recherches environnementales visant à restituer la topographie et l'évolution des paysages agraires.

INVENTAIRE DES SITES (fig. 21)

(liste établie en 1999)

\section{BOUCHES-DU-RHÔNE}

\section{AIX-En-Provence}

\section{ZAC Sextius-Mirabeau}

Site : coteau situé au sud de la ville antique.

Type d'opération: fouille préventive.

Type de plantation: fosses allongées (type 4).

Contexte archéologique et dispositifs associés: dans un enclos funéraire abandonné ?

Datation: II ${ }^{\mathrm{e}} \mathrm{s}$. de notre ère

Bibliographie: Nin, Vecchione, 1998, p. 64.

\section{Signoret}

Site: coteau au nord de l'agglomération antique, au pied de la colline Saint-Eutrope.

Type d'opération: fouille préventive préalable à la construction d'un parking souterrain (dir. L. Rivet).

Type de plantation : traces perpendiculaires correspondant peut-être à des tranchées.

Contexte archéologique et dispositifs associés: au sein d'un quartier artisanal et devant un bâtiment.

Datation: Haut-Empire.

Bibliographie: Rivet, 1992. 


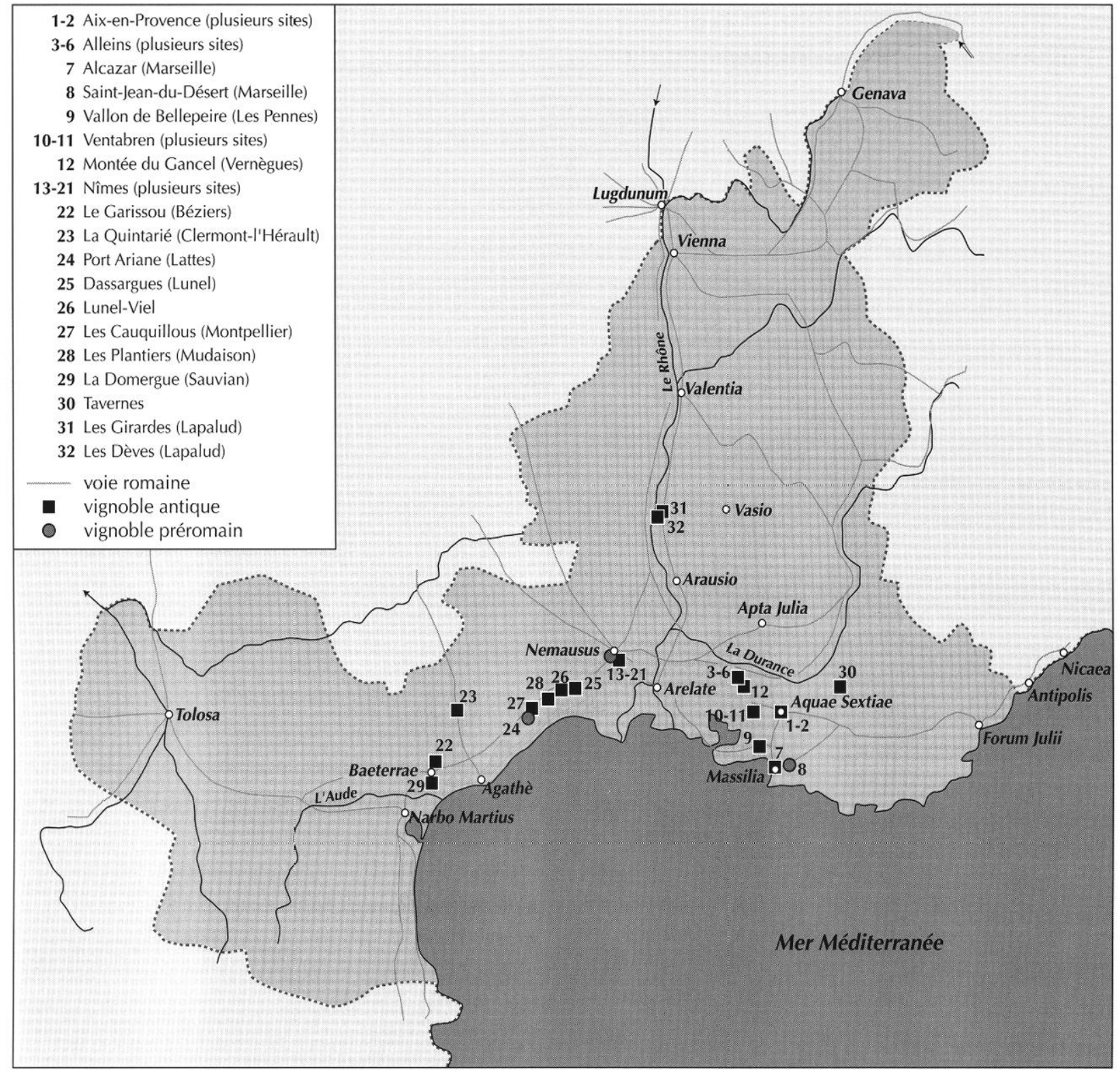

Fig. 21 - Carte des principaux sites ayant liuré des indices de vignobles en Narbonnaise (période préromaine el romaine).

\section{AI.I.EINS}

\section{Derrière Saint-Pierre}

Site: coteau au piémont nord d'un massif calcaire.

Type d'opération : fouille préventive dans le cadre de l'opération TGV-Méditerranée (dir. P. Boissinot).

Type de plantation: fosses allongées (type 4) et tranchées (type 1).

Contexte archéologique et dispositifs associés: aucun, si ce n'est la présence d'un fossé au nord.
Datation: Antiquité (sans plus de précision). Bibliographie : Boissinot, 1997.

\section{Jardinets-Pierrefeu}

Site: ancienne terrasse dominant le vallon de Tamberlette.

Type d'opération : fouille préventive dans le cadre de l'opération TGV-Méditerranée (dir. L. Cordier).

Type de plantation: tranchées étroites (type 1), parfois regroupées par trois (culture de la vigne possible). 
Contexte archéologique et dispositifs associés: murs, fosses et foyers antiques mis en évidence à proximité.

Datation: Haut-Empire.

Bibliographie: inédit (rens. L. Cordier).

\section{Jonquerolles}

Site : coteau exposé au nord.

Type d'opération : fouille préventive dans le cadre de l'opération TGV-Méditerranée (dir. P. Chapon).

Type de plantation: fosses allongées (type 4).

Contexte archéologique et dispositifs associés: présence de plantations d'arbres?

Datation: Antiquité.

Bibliographie: inédit (rens. P. Chapon).

\section{Tamberlette}

Site: dans un vallon au pied d'une colline.

Type d'opération: fouille préventive dans le cadre de l'opération TGV-Méditerranée (dir. J.-P. Pillard).

Type de plantation: fosses rondes (type 2) et allongées (type 4).

Contexte archéologique et dispositifs associés: dans la proche périphérie d'une villa.

Datation: I ${ }^{\text {er }}$ s. aprc̀s J.-C.

Bibliographie: Pillard, Almès, 1998.

\section{Marseille}

\section{Alcazar}

Site: piémont d'une butte à l'est de la ville antique.

Type d'opération: fouille préventive liée à la construction d'une médiathèque (dir. M. Bouiron).

Type de plantation: tranchées (type 1).

Contexte archéologique el disposilifs associés: ?

Datation: Antiquité tardive.

Bibliographie: inédit (rens. M. Bouiron).

\section{Saint-Jean-du-Désert}

Site: fond de vallon.

Type d'opération : fouille préventive dans le cadre de l'opération Rocade L2 (dir. P. Boissinot).
Type de plantation: tous les types sont représentés (1 à 4). Contexte archéologique et dispositifs associés: le long d'un ruisseau et suivant un découpage en lanières, hors de tout contexte d'habitat.

Datation: IV $^{\mathrm{e}}-\mathrm{II}^{\mathrm{e}} \mathrm{s}$. avant J.-C. pour la partie la mieux conservée.

Bibliographie : Boissinot, 1995b, 1997 ; Boissinot, Brochier, 1997 ; Boissinot, Sauvage, 2000.

\section{Pennes-Mirabeau (Les)}

\section{Vallon de Bellepeire}

Site: fond de vallon.

Iype d'opération: fouille préventive dans le cadre de l'opération TGV-Méditerranée (dir. P. Boissinot).

Type de plantation: tranchées (type 1).

Contexte archéologique et dispositifs associés: ?

Datation: Antiquité tardive?

Bibliographie: Boissinot, 1997.

\section{VENTABREN}

\section{Les Bosques}

Site: coteau exposé au nord.

Type d'opération : fouille préventive dans le cadre de l'opération TGV-Méditerranée (dir. P. Boissinot).

Type de plantation: tranchées (type 1) et fosses allongées (type 4).

Contexte archéologique et dispositifs associés : ?

Datation: Haut-Empire.

Bibliographie: Boissinot, 1997.

\section{Château-Blanc}

Site : coteau au pied d'un massif calcaire.

Type d'opération: fouille préventive dans le cadre de l'opération TGV-Méditerranée (dir. P. Boissinot).

Type de plantation: fosses allongées (type 4).

Contexte archéologique et dispositifs associés: à proximité d'une villa repérée en photographie aérienne.

Datation : Haut-Empire.

Bibliographie: Boissinot, 1997 et à paraître. 


\section{VERNÈGUES}

\section{Montée du Gancel}

Site: coteau exposé au nord.

Type d'opération: fouille préventive dans le cadre de l'opération TGV-Méditerranée (dir. P. Boissinot).

Type de plantation: fosses allongées (type 4).

Contexte archéologique el dispositifs associés: champ inscrit dans un parcellaire souligné par des murs à proximité de l'agglomération antique de Château-Bas (Vernègues).

Datation: Haut-Empire.

Bibliographie: Boissinot, Brochier, 1997.

\section{GARD}

\section{NîMES}

\section{Le Florian}

Site: piémont d'une colline en rive gauche d'un cours d'eau intermittent à la périphérie de la ville romaine.

Type d'opération: fouille préventive préalable à la construction d'une HLM.

Type de plantation: tranchécs (type 1), fosses ramassées (type 3) et fosses allongées (type 4).

Contexte archéologique et dispositifs associés: périphérie urbaine.

Datation: II' et $\mathrm{I}^{\mathrm{er}}$ s. avant J-C.

Bibliographie: Monteil et al., 1999.

\section{4. Îlot Thérond}

Site: plaine, au bas d'un versant exposé à l'est.

Type d'opération: fouille préventive (dir. L. Sauvage).

Type de plantation: fosses circulaires de petite taillc (type 2) et fosses allongées (type 4).

Conlexle archéologique et dispositifs associés: espace exclusivement agricole à cette période.

Datation: $\mathrm{I}^{\mathrm{er}} \mathrm{s}$. avant J.-C.

Bibliographie: Monteil et al., 1999.

\section{5. Îlot Grill}

Site : plaine.

Type d'opération: fouille préventive (dir. L. Duflot).
Type de plantation: tranchées (type 1).

Contexte archéologique et dispositifs associés: ? (sondage ponctuel).

Datation: période républicaine.

Bibliographie: Monteil el al., 1999.

\section{Rue Émile-Jamais}

Site: flanc de colline.

Type d'opération: fouille préventive (dir. M.-L. Hervé).

Type de plantation: petites fosses allongées (type 4) avec indices de provignage.

Conlexte archéologique et dispositifs associés: présence d'un fossé ou d'une dépression artificielle.

Datation: transition $\mathrm{II}^{\mathrm{C}}-\mathrm{I}^{\mathrm{er}}$ s. avant J.-C.

Bibliographie: Monteil et al., 1999.

\section{ZAC des Halles}

Site: frange méridionale d'une vaste cuvette humide, en bordure de piémont.

Type d'opération: fouille préventive (dir. M. Monteil).

Type de plantation: fosses allongées (type 4).

Contexte archéologique et dispositifs associés: aménagements agraires complexes, avec indices d'irrigation et réalisation de terrasses à la périphérie de la ville préromaine.

Datation: II ${ }^{\mathrm{e}}$ s. avant J.-C.

Bibliographie: Monteil dir., 1993.

\section{ZAC du Forum des Halles}

Sile : versant interrompu par un talweg.

Type d'opération : fouille préventive (dir. V. Bel).

Type de plantation: tranchées (type 1) et fosses ramassées (type 3).

Contexle archéologique et dispositifs associés: bordure d'une voic cn crcux comportant une tombe.

Datation: II $\mathrm{e}$ s. avant J.-C.-- ${ }^{\mathrm{er}}$ s. après J.-C.

Bibliographie: Monteil el al., 1999.

\section{Saint-André-de-Codols}

Site: plaine du Vistre.

Type d'opération: fouille préventive (dir. L. Vidal).

Type de plantation: tranchées (type 1).

Contexte archéologique et dispositifs associés: proximité d'une villa et d'un ensemble de fossés. 
Datation: Haut-Empire.

Bibliographie: Vidal, 1997.

\section{Le Viol-du-Plan}

Site : plaine du Vistre.

Type d'opération: fouille préventive (dir. L. Vidal).

Type de plantation: tranchées (type 1).

Contexte archéologique et dispositifs associés : réseau de fossés.

Datation: époque républicaine.

Bibliographie: Vidal, Petitot, 1992 ; Vidal, 1997.

\section{Le Mas Carbonnel}

Site : plaine du Vistre.

Type d'opération: fouille préventive (dir. H. Petitot et L. Sauvage).

Type de plantation: tranchées (type 1) et fosses allongées (type 4).

Contexte archéologique et dispositifs associés : fossés.

Datation: II ${ }^{\mathrm{e}}$ s. avant J.-C. et Haut-Empire.

Bibliographie: Vidal, Petitot, 1992 ; Vidal, 1997.

\section{HÉRAULT}

\section{BÉZIERS}

\section{Le Garissou}

Site: flanc de colline à $5 \mathrm{~km}$ au nord-est de la ville antique.

Type d'opération: fouille préventive sur le tracé de la rocade nord de Béziers (dir. N. Lecuyer).

Type de plantation: fosses allongées (type 4).

Contexte archéologique et dispositifs associés: à proximité d'une villa.

Datation: Antiquité.

Bibliographie : Lecuyer, 1992.

\section{Clermont-L'Hérault}

\section{La Quintarié}

Site: terrasse ancienne de l'Hérault, au pied des collines miocènes.

Type d'opération : fouille préventive dans le cadre de l'opé- ration A 75 (dir. P. Boissinot).

Type de plantation: fosses allongées (type 4).

Contexte archéologique et dispositifs associés: dans un parcellaire complexe et à proximité d'une ferme-relais située au carrefour de deux voies.

Datation: Haut-Empire.

Bibliographie: Boissinot, à paraître.

\section{LATTES}

\section{Port Ariane}

Site : plaine alluviale en bordure du Lez.

Type d'opération: fouille de sauvetage liée à l'aménagement d'un port fluvial (dir. L. Jallot, étude d'impact).

Type de plantation: fosses allongées (type 4) avec indices de provignage.

Contexte archéologique et dispositifs associés: ?, fouille en cours.

Datation: second âge du Fer.

Bibliographie: inédit (rens. L. Jallot et C. Richarté).

\section{LUNEL}

\section{Dassargues}

Site: dans la plaine alluviale du Vidourle.

Type d'opération: fouille préventive préalable à la construction d'un centre commercial et d'un carrefour routier (dir. C. Raynaud).

Type de plantation: fosses allongées (type 4) avec indices de provignage.

Contexte archéologique et dispositifs associés: dans un parcellaire complexe délimité par des fossés.

Datation: Haut-Empire.

Bibliographie: Favory et al., 1993 ; Garnier et al., 1995; Favory, 1997.

LUNEL-VIEL

\section{Village}

Site : plaine littorale.

Type d'opération: sauvetage programmé (dir. C. Raynaud). Type de plantation: fosses allongées (type 4).

Contexte archéologique et dispositifs associés : zone agraire du quartier central de l'agglomération antique. 
Datation: Haut-Empire.

Bibliographie: Raynaud, 1990 ; Favory et al., 1993.

\section{MONTPELLIER}

\section{Les Cauquillous}

Site : plaine littorale au sud de Montpellier.

Type d'opération: fouille préventive préalable à l'installation d'un complexe de cinémas (dir. L. Sauvage).

Type de plantation: fosses de petite taille (type 2) avec indices de provignage.

Contexte archéologique et dispositifs associés: au sein d'un ensemble fossoyé, en bordure d'un chemin empierré et à proximité d'un ensemble fossoyé (funéraire ?) du premier âge du Fer.

Datation : au plus tôt, au $\mathrm{I}^{\mathrm{er}}$ s. avant J.-C.

Bibliographie: Dedet, Sauvage, 1998.

\section{MUDAISON}

\section{Les Plantiers}

Site: sur une légère éminence, en bordure de la vallée du Bérange, dans la plaine littorale.

Type d'opération: fouille de sauvetage (dir. K. Roger) à l'occasion de travaux agricoles (défonçage).

Type de plantation: "saignées de culture ".

Contexte archéologique et dispositifs associés: ensemble de fossés et de fosses, dont l'une a certainement contenu un dolium.

Datation: I $^{\mathrm{er}}-\mathrm{II}{ }^{\mathrm{c}} \mathrm{s}$. après J.-C.

Bibliographie: Favory et al., 1993.

\section{SAUVIAN}

\section{I a Domergue}

Site: plaine alluviale de l'Orb à $5 \mathrm{~km}$ au sud-est de Béziers.

Type d'opération: fouille préventive à l'emplacement d'une future ZAC.

Type de plantation: tranchées (type 1).

Contexte archéologique et dispositifs associés: à proximité d'une villa, dans un contexte de fossés et de chemins.
Datation: Haut-Empire.

Bibliographie: Vidal, 1998.

\section{VAR}

\section{TAVERNES}

\section{Roure-Gros}

Site : plaine.

Type d'opération: diagnostic.

Type de plantation: fosses allongées (type 4).

Contexte archéologique et dispositifs associés: ?

Datation: Antiquité ?

Bibliographie : inédit (rens. L. Martin).

\section{VAUCLUSE}

\section{LAPALUD}

\section{Les Girardes}

Site: plaine du Tricastin.

Type d'opération : fouille préventive dans le cadre du TCVMéditerranée (emprunt de matériaux) (dir. P. Boissinot et K. Roger).

Type de plantation : fosses rectangulaires, carrées et rondes (types 2, 3 et 4).

Contexte archéologique et dispositifs associés: à proximité d'une ferme et dans le cadre du cadastre B d'Orange.

Datation: Haut-Empire.

Bibliographie: Boissinot, 1997 ; Boissinot, Brochier, 1997.

\section{Les Dèves}

Site : plaine du Tricastin.

Type d'opération : fouille préventive dans le cadre du TGVMéditerranée (dir. A. Gelot).

Type de plantation: fosses carrées (type 3).

Contexte archéologique et dispositifs associés: ensemble fossoyé.

Datation: Haut-Empire.

Bibliographie: DFS inédit. 